Pacific

Journal of

Mathematics

THE UNITARY DUAL OF THE HERMITIAN QUATERNIONIC GROUP OF SPLIT RANK 2

MARCELA HANZER 


\title{
THE UNITARY DUAL OF THE HERMITIAN QUATERNIONIC GROUP OF SPLIT RANK 2
}

\author{
MARCELA HANZER
}

\begin{abstract}
We classify the irreducible noncuspidal representations of the hermitian quaternionic group of split rank 2. We also find the complete noncuspidal unitary dual of this nonquasisplit group.
\end{abstract}

\section{Introduction and preliminaries}

We are interested in studying the composition series and unitary dual of the $p$-adic hermitian quaternionic group of semisimple rank 2 , denoted by $G_{2}(D)$. This group is not quasisplit, so the calculations of the unitary dual cannot be obtained by the direct application of Shahidi's methods. The group has an interesting feature: it has an isolated unitary representation, a phenomenon that occurs, for example, in the case of the exceptional group $G_{2}$.

We also calculate the unitary dual supported on the non-Siegel maximal parabolic subgroup using global methods similar to those used in [Muić and Savin 2000] for the Siegel case, but resolving some obstacles related to the Langlands correspondence between the hermitian quaternionic group of semisimple rank 1 and its split form. Similar classifications were obtained for classical split groups by Sally and Tadić [1993] for $p$-adic $\operatorname{GSp}(2, F)$ and $\operatorname{Sp}(2, F)$, and by Konno [2001] for the quasisplit unitary group. Regarding the exceptional groups, the classification for the group $G_{2}$ was done by Muić [1997]. In the classification of the subquotients of the principal series of the hermitian quaternionic group we use the structure of the $\Psi$-Hopf module on the Grothendieck group of the representations of the finite length. This structure in the case of the split connected groups with the root system of types $C_{n}$ and $B_{n}$ was observed by Tadić [1995] and then, in the case of $O(2 n, F)$, the similar result was obtained by Ban [1999].

In this section, we recall the structure of the hermitian quaternionic groups, state a result about the aforementioned structure of the $\Psi$-Hopf module on the Grothendieck group, and state the Langlands' classification and the criterion for square integrability. We resolve the questions of the reducibility of the induced

MSC2000: 22E35.

Keywords: unitary dual, $p$-adic groups, hermitian quaternionic groups. 
representations for the hermitian quaternionic group of the semisimple rank 1 and make some observations about its structure.

In Section 2 we deal with the reducibility and composition series for the principal series for the group $G_{2}(D)$. Sections 3 and 4 are devoted to the determination of reducibility in the case of the induction from the maximal parabolic subgroups. Section 5 is devoted to the classification of the noncuspidal part of the unitary dual of the group $G_{2}(D)$.

For the admissible representation $\sigma$ of any group we consider, we denote by $\omega_{\sigma}$ its central character (if it exists). We will denote the Steinberg representation of the group $G$ by $S t_{G}$. If $H$ is a subgroup of the group $G$ and $g \in G$ normalizes $H$, for the representation $\sigma$ of the group $H$, we denote by ${ }^{g} \sigma$ the representation of the group $H$ defined by ${ }^{g} \sigma(h)=\sigma\left(g^{-1} h g\right)$. We denote by $\{\alpha, \beta\}$ the basis of the root system corresponding to the maximal $F$-split torus in $G_{2}(D)$. The choice of the maximal $F$-split torus will be given in the next subsection. Also $\{\alpha, \beta\}$ will denote the basis of the root system with respect to the diagonal subgroup in $\operatorname{SO}(4, F)$.

Hermitian quaternionic groups. Let $F$ be a nonarchimedean local field of characteristic zero, having residual field with $q$ elements. We choose a uniformizer of the field and denote it by $\bar{\omega}$. Let $D$ be a quaternionic algebra central over $F$ and let $\tau$ be an involution (of the first kind) fixing the center of $D$. By [Mœglin et al. 1987], the division algebra $D$ defines a reductive group $G$ over $F$ as follows. Let

$$
V_{n}=e_{1} D \oplus \cdots \oplus e_{n} D \oplus e_{n+1} D \oplus \cdots \oplus e_{2 n} D
$$

be a right vector space over $D$. The relations $\left(e_{i}, e_{2 n-j+1}\right)=\delta_{i j}$ for $i=1,2, \ldots, n$ define a hermitian form on $V_{n}$ :

$$
\begin{aligned}
\left(v, v^{\prime}\right) & =\varepsilon \tau\left(\left(v^{\prime}, v\right)\right) & & \text { for } v, v^{\prime} \in V_{n}, \varepsilon \in\{-1,1\}, \\
\left(v x, v^{\prime} x^{\prime}\right) & =\tau(x)\left(v, v^{\prime}\right) x^{\prime} & & \text { for } x, x^{\prime} \in D .
\end{aligned}
$$

We extend the involution $\tau$ on $M(k, D)$, denoting it by *

$$
g^{*}=\left(g_{i j}\right)^{*}=\tau\left(g_{i j}\right)^{t} .
$$

For a smooth representation $\tau$ of the group $\operatorname{GL}(n, D)$, we define the representation

$$
\tau^{*}(g)=\tau\left(g^{-*}\right) .
$$

By the observation in [Muić and Savin 2000], for the irreducible smooth representation $\tau$ of the group $\operatorname{GL}(\cdot, D)$, the relation $\tau^{*} \cong \tilde{\tau}$ holds. Let $G_{n}(D, \varepsilon)$ be the group of the isometries of the form $(\cdot, \cdot)$. We can also describe $G_{n}(D)$ as

$$
G_{n}(D)=\left\{g \in \mathrm{GL}(2 n, D): g^{*}\left(\begin{array}{cc}
0 & J_{n} \\
-J_{n} & 0
\end{array}\right) g=\left(\begin{array}{cc}
0 & J_{n} \\
-J_{n} & 0
\end{array}\right)\right\}
$$


where $J_{n}=\left(\begin{array}{c}. \\ \end{array}{ }^{1}{ }^{1}\right)$
\[ D \otimes_{F} \bar{F} \cong M(2, \bar{F}), \]

and the well-known explicit matrix realization of the algebra $D$, it is easy to see that $G_{n}(D, \varepsilon)$ is the group of $F$-rational points of a reductive algebraic group, an inner form of the split group $\mathrm{SO}(4 n)$ or $\operatorname{Sp}(4 n)$, if $\varepsilon=-1$ or $\varepsilon=1$, respectively. In this paper, we will do explicit calculations for the case $\varepsilon=-1$, so we drop $\varepsilon$ from the notation and, unless otherwise specified, assume $\varepsilon=-1$. Having in mind a matrix representation of the group of $\bar{F}$-rational points, there is a maximal (diagonal) split torus $A_{0}$ which has the following set of $F$-rational points:

$$
A_{0}(F)=\left\{\left(\begin{array}{ccccccc}
\lambda_{1} & & & & & & \\
& \lambda_{2} & & & & & \\
& & \ddots & & & & \\
& & & \lambda_{n} & & & \\
& & & \lambda_{n}^{-1} & & & \\
& & & & \ddots & & \\
& & & & & \lambda_{2}^{-1} & \\
& & & & & & \lambda_{1}^{-1}
\end{array}\right): \lambda_{i} \in F^{*}\right\} .
$$

The element of $A_{0}(F)$ shown is denoted by $\operatorname{diag}\left(\lambda_{1}, \ldots, \lambda_{n}\right) . X\left(A_{0}\right)$ denotes the group of $F$-rational characters on $A_{0}$, which can be identified with characters on $A_{0}(F)$. The root system corresponding to the maximal $F$-split torus is of type $C_{n}$, with the set of simple roots $\left\{\alpha_{i}, i=1, \ldots, n\right\}$, where

$$
\begin{aligned}
& \alpha_{i}\left(\operatorname{diag}\left(\lambda_{1}, \ldots, \lambda_{n}\right)\right)=\lambda_{i} \lambda_{i+1}^{-1}, \quad \text { for } i=1, \ldots, n-1, \\
& \alpha_{n}\left(\operatorname{diag}\left(\lambda_{1}, \ldots, \lambda_{n}\right)\right)=\lambda_{n}^{2} .
\end{aligned}
$$

The standard Levi $F$-subgroups correspond to the subsets $\theta$ of the set of simple roots $\Delta=\Delta\left(G_{n}(D), A_{0}\right)$ in the root system and we denote them by $M_{\theta}$. To describe the Levi subgroups $M_{\theta}(F)$, we set $B_{l}=J_{l}\left(A_{l}^{-1}\right)^{*} J_{l}$ if $A_{l}$ is a quadratic matrix of order $l$.

(i) If $\alpha_{n} \notin \theta$ there are positive integers $n_{1}, n_{2}, \ldots, n_{k}$ such that $\sum n_{i}=n$ and

$$
M_{\theta}(F)=\left\{\left(\begin{array}{cccccc}
A_{n_{1}} & & & & & \\
& \ddots & & & & \\
& & A_{n_{k}} & & & \\
& & & B_{n_{k}} & & \\
& & & & \ddots & \\
& & & & & B_{n_{1}}
\end{array}\right): A_{n_{i}} \in \mathrm{GL}\left(n_{i}, D\right)\right\} .
$$


(ii) If $\alpha_{n} \in \theta$ there are positive integers $n_{1}, n_{2}, \ldots, n_{k}, r$ such that $\sum n_{i}+r=n$ and

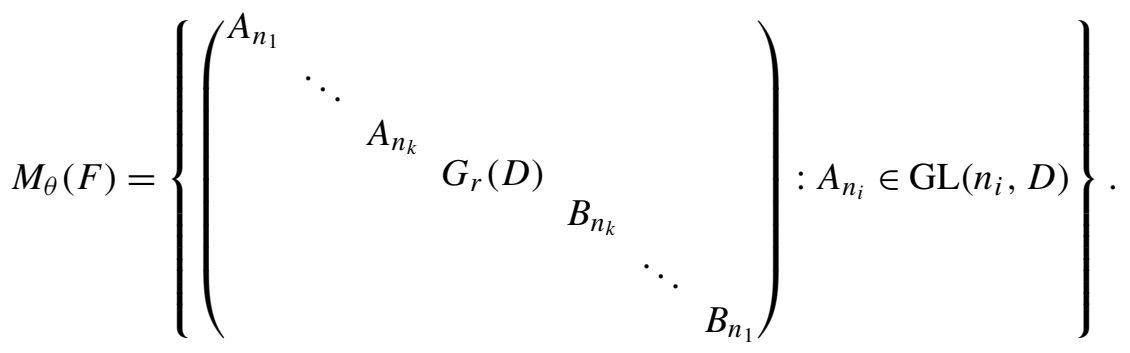

We describe the Langlands classification, following [Borel and Wallach 2000]. Let $v(x)=|x|_{F}$ if $x \in F$ and $v(x)=|\operatorname{det} x|_{F}$ if $x \in D$; here det is the norm homomorphism. For any essentially square integrable representation (mod center) $\delta$ of the group $\operatorname{GL}(m, D)$, there exists a unique real number $e(\delta)$ and a unique square-integrable representation $\delta^{u}$ such that $\delta=v^{e(\delta)} \delta^{u}$. We say that an (ordered) multiset $\left(\delta_{1}, \delta_{2}, \ldots, \delta_{k}\right)$ of irreducible essentially square-integrable representations of GL( $(\cdot, D)$-groups is in standard order if $e\left(\delta_{1}\right) \geq e\left(\delta_{2}\right) \geq \cdots \geq e\left(\delta_{k}\right)$. For the representations $\delta_{i}$ of $\operatorname{GL}(\cdot, D)$ groups and representation $\tau$ of the group $G_{r}(D)$, we write

$$
\delta_{1} \times \delta_{2} \times \cdots \times \delta_{k} \rtimes \tau=\operatorname{Ind}_{P}^{G_{n}(D)}\left(\delta_{1} \otimes \delta_{2} \otimes \cdots \otimes \delta_{k} \otimes \tau\right),
$$

where $P$ is a corresponding standard parabolic subgroup of $G_{n}(D)$. Suppose $\left(\delta_{1}, \delta_{2}, \ldots, \delta_{k}\right)$ is a multiset of irreducible essentially square-integrable representations of $\mathrm{GL}(\cdot, D)$-groups which is in the standard order, and assume that $e\left(\delta_{k}\right)>0$. If $\tau$ is an irreducible tempered representation of $G_{r}(D)$, we consider the representation $\delta=\delta_{1} \otimes \delta_{2} \otimes \cdots \otimes \delta_{k} \otimes \tau$ of the corresponding standard Levi subgroup and let

$$
e(\delta)=\left(e\left(\delta_{1}\right), e\left(\delta_{1}\right), \ldots, e\left(\delta_{k}\right), e\left(\delta_{k}\right), 0, \ldots, 0\right) \in X\left(A_{0}\right) \otimes_{\mathbb{Z}} \mathbb{R} \cong \mathbb{R}^{n} .
$$

Here the number $e\left(\delta_{i}\right)$ appears in $e(\delta)$ exactly $n_{i}$ times if $\delta_{i}$ is a representation of the group $\mathrm{GL}\left(n_{i}, D\right)$, and 0 appears $r$ times. We introduce a partial order on $X\left(A_{0}\right) \otimes_{\mathbb{Z}}$ $\mathbb{R} \cong \mathbb{R}^{n}$ related to the root system of type $\mathrm{C}_{\mathrm{n}}$. We say that $\left(x_{1}, x_{2}, \ldots, x_{n}\right) \leq$ $\left(y_{1}, y_{2}, \ldots, y_{n}\right)$ if and only if $\sum_{i=1}^{k} x_{i} \leq \sum_{i=1}^{k} y_{i}$ for $k=1, \ldots, n$. This order is the one obtained in general as follows. Let $(\cdot, \cdot)$ be the Weyl group-invariant scalar product on $X\left(A_{0}\right) \otimes \mathbb{R}$ and let $\left(\beta_{1}, \beta_{2}, \ldots, \beta_{n}\right)$ be the basis bidual to $\left(\alpha_{1}, \ldots, \alpha_{n}\right)$. Then for $v_{1}, v_{2} \in X\left(A_{0}\right) \otimes_{\mathbb{Z}} \mathbb{R}$ we say $v_{1} \leq v_{2}$ if and only if $\left(v_{1}, \beta_{i}\right) \leq\left(v_{2}, \beta_{i}\right)$ for all $i=1, \ldots, n$.

Lemma 1.1 (Langlands' classification [Borel and Wallach 2000]). The induced (standard) representation $\delta_{1} \times \delta_{2} \times \cdots \times \delta_{k} \rtimes \tau$, where the irreducible essentially square-integrable representations $\left(\delta_{1}, \delta_{2}, \ldots, \delta_{k}\right)$ are in the standard order with $e\left(\delta_{k}\right)>0$ and where $\tau$ is an irreducible tempered representation of the group 
$G_{r}(D)$, has a unique irreducible quotient, denoted $L\left(\delta_{1}, \delta_{2}, \ldots, \delta_{k} ; \tau\right)$, which is of the multiplicity one in the induced representation. In this way, we obtain every irreducible representation of the group $G_{n}(D)$. If the standard representation $\delta_{1} \times \delta_{2} \times \cdots \times \delta_{k} \rtimes \tau$ has an irreducible subquotient $\sigma=L\left(\delta_{1}^{\prime}, \ldots, \delta_{k^{\prime}} ; \tau^{\prime}\right)$ other than its Langlands quotient, then $e\left(\delta_{1}^{\prime} \otimes \delta_{2}^{\prime} \otimes \cdots \otimes \delta_{k^{\prime}}^{\prime} \otimes \tau^{\prime}\right)<e\left(\delta_{1} \otimes \delta_{2} \otimes \cdots \delta_{k} \otimes \tau\right)$.

Given an admissible irreducible representation $\pi$ of $G_{n}(D)$ and an ordered partition $\alpha=\left(n_{1}, n_{2}, \ldots, n_{k}\right)$ of $n-r$, let $s_{(\alpha)}(\pi)$ denote the normalized Jacquet module of $\pi$ with respect to the standard parabolic subgroup $P_{\alpha}$ with Levi subgroup isomorphic to $\mathrm{GL}\left(n_{1}, D\right) \times \mathrm{GL}\left(n_{2}, D\right) \times \cdots \times \mathrm{GL}\left(n_{k}, D\right) \times G_{r}(D)$. Let $P_{\alpha}$ denote a standard parabolic subgroup minimal with the property that $s_{(\alpha)}(\pi) \neq 0$. Each irreducible subquotient of $s_{(\alpha)}(\pi)$ is necessarily cuspidal. The square integrability criterion from [Casselman 1995] for general $p$-adic reductive groups readily applies, and we obtain:

Lemma 1.2 (Square integrability criterion). A necessary and sufficient condition for an irreducible admissible representation $\pi$ to be square-integrable is that, for every ordered partition $\alpha=\left(n_{1}, n_{2}, \ldots, n_{k}\right)$ of $n-r$ minimal with the property $s_{\alpha}(\pi) \neq 0$ and every irreducible subquotient subquotient $\sigma$ of $s_{(\alpha)}(\pi)$, we have

$$
\left(e(\sigma), \beta_{n_{1}+\cdots+n_{i}}\right)>0 \text { for all } i=1, \ldots, k \text {. }
$$

Given an admissible representation $\sigma$ of the standard Levi subgroup $M_{\theta}$ and an element $w$ of the Weyl group such that $w(\theta)=\theta^{\prime}$ is subset of the set of the simple roots, we set $N_{w}=N_{0} \cap w \bar{N}_{\theta} w^{-1}$, where $\bar{N}_{\theta}$ is the unipotent radical of the parabolic subgroup opposite to $P_{\theta}$. For $m \in M_{\theta^{\prime}}$ we define the representation of $M_{\theta^{\prime}}$ by ${ }^{w} \sigma(m)=\sigma\left(w^{-1} m w\right)$. We define (formally), for $f \in \operatorname{Ind}_{M_{\theta}}^{G_{n}(D)}(\sigma)$,

$$
A_{w}(\sigma) f(g)=\int_{N_{w}} f\left(w^{-1} n g\right) d n .
$$

If this integral converges for every $f$, it defines the intertwining operator

$$
A_{w}(\sigma): \operatorname{Ind}_{M_{\theta}}^{G_{n}(D)}(\sigma) \rightarrow \operatorname{Ind}_{M_{\theta^{\prime}}}^{G_{n}(D)}\left({ }^{w} \sigma\right) .
$$

Often, the operator $A_{w}$ will have some additional (complex) arguments, usually denoting the action of the family of intertwining operators on the family of the representations, which depends on these complex numbers in an obvious way. If $w$ is the longest element in the relative Weyl group, we call the operator $A_{w}$ the long intertwining operator. Sometimes we use a different definition for the long intertwining operator: we denote by $\delta_{1} \underline{\times} \delta_{2} \underline{\times} \cdots \underline{x} \delta_{k} \underline{\rtimes} \tau$ the representation of $M_{\theta}$ induced from the opposite (lower-triangular) parabolic subgroup. The longintertwining operator from the representation space of the standard representation 
$\delta_{1} \times \delta_{2} \times \cdots \times \delta_{k} \rtimes \tau$ to the representation space of the representation $\delta_{1} \underline{\times} \delta_{2} \underline{\times}$ $\cdots \times \delta_{k} \unlhd \tau$ is denoted by $R$ and defined (formally) by

$$
R\left(\delta_{1} \otimes \delta_{2} \otimes \cdots \otimes \delta_{k} \otimes \tau, N_{\theta}, \bar{N}_{\theta}\right) f(g)=\int_{\bar{N}_{\theta}} f(\bar{n} g) d \bar{n} .
$$

If this operator is injective or surjective (for the standard representation $\delta_{1} \times \delta_{2} \times$ $\left.\cdots \times \delta_{k} \rtimes \tau\right)$, the representation $\delta_{1} \times \delta_{2} \times \cdots \times \delta_{k} \rtimes \tau$ is irreducible.

Reducibility of the induced representations. We recall briefly some results from [Bernstein et al. 1984; Tadic 1990] about the reducibility of the induced representations of $\mathrm{GL}(n, D)$. To the irreducible cuspidal representation $\sigma$ of the group $\mathrm{GL}(n, D)$, Jacquet-Langlands correspondence attaches an irreducible essentially square-integrable representation $\sigma^{\prime}$ of the group $\operatorname{GL}(2 n, F)$. If $\sigma^{\prime}$ is a cuspidal representation, we set $s(\sigma)=1$, and if $\sigma^{\prime}$ is a subquotient of the induced representation $\tau \times \tau \nu$ for some irreducible cuspidal representation $\tau$ of the group $\operatorname{GL}(n, F)$, we set $s(\sigma)=2$. These are the only possibilities. We then set $v_{\sigma}=v^{s(\sigma)}$. Then, for the irreducible cuspidal representations $\sigma_{i}$ of $\operatorname{GL}\left(n_{1}, D\right)$ and $\operatorname{GL}\left(n_{2}, D\right)$, the representation $\sigma_{1} \times \sigma_{2}$ is reducible if and only if $n_{1}=n_{2}, s\left(\sigma_{1}\right)=s\left(\sigma_{2}\right)$ and $\sigma_{1}=v_{\sigma_{2}}^{ \pm 1} \sigma_{2}$.

Using the factorization of the long intertwining operator [Speh and Vogan 1980] we obtain the following lemma, for which see also [Tadić 1994].

Lemma 1.3 (Reducibility of the principal series). For the irreducible admissible representations $\tau_{i}$ of the $D^{*}$ the principal series representation $\tau_{1} \times \cdots \times \tau_{n} \rtimes 1$ of the group $G_{n}(D)$ reduces if and only if

(i) there exists $i$ such that $\tau_{i} \rtimes 1$ or $\tilde{\tau}_{i} \rtimes 1$ reduces in $G_{1}(D)$, or

(ii) there exist distinct $i$ and $j$ such that $\tau_{i} \times \tau_{j}$ or $\tilde{\tau}_{i} \times \tau_{j}$ or $\tau_{i} \times \tilde{\tau}_{j}$ or $\tilde{\tau}_{i} \times \tilde{\tau}_{j}$ reduce in $\mathrm{GL}(2, D)$.

We will describe reducibility in $G_{1}(D)$ shortly.

We recall from [Zelevinsky 1981; Tadic 1990] the Hopf algebra structure on the Grothendieck group $R_{n}$ of smooth representations of finite length of the group $\mathrm{GL}(n, D)$. Let $R(*)$ be the Grothendieck group related to the corresponding reductive group, and $R=\bigoplus_{n \geq 0} R_{n}$. The multiplication $m: R \otimes R \rightarrow R$ is defined by induction, and comultiplication $m^{*}: R \rightarrow R \otimes R$ by Jacquet modules:

$$
m^{*}(\pi)=\sum_{k=0}^{n} s . s\left(r_{(k, n-k),(n)}(\pi)\right) \in R \otimes R .
$$

Here $\pi$ is a smooth representation of finite length of $\operatorname{GL}(n, D)$, and $r_{(k, n-k),(n)}(\pi) \in$ $R_{k} \otimes R_{n-k}$ is the normalized Jacquet module with respect to the maximal standard parabolic subgroup with Levi subgroup $\mathrm{GL}(k, D) \times \mathrm{GL}(n-k, D)$. By linearity, we 
extend the definition of $m$ and $m^{*}$ to $R$. The tensor product $R \otimes R$ has an algebra structure in the usual way. The comultiplication $m^{*}$ is a ring homomorphism; the proof can be found in [Zelevinsky 1981] for the case of general linear groups over the field $F$.

Set $R(G)=\bigoplus_{n \geq 0} R\left(G_{n}(D)\right)$. This is obviously and $R$-module, and a comodule structure is defined like the one in the GL-case: for a smooth, finite length representation $\pi$ of the group $G_{n}(D)$ we put

$$
\mu^{*}(\sigma)=\sum_{k=0}^{n} s . s\left(s_{(k)}(\sigma)\right) .
$$

Denote by $s: R \otimes R \rightarrow R \otimes R$ the linear map such that $s\left(\pi_{1} \otimes \pi_{2}\right)=\pi_{2} \otimes \pi_{1}$ for representations $\pi_{1}$ and $\pi_{2}$. Define the ring homomorphism $\Psi: R \rightarrow R \otimes R$ by

$$
\Psi=(m \otimes 1) \circ\left(\sim \otimes m^{*}\right) \circ s \circ m^{*} .
$$

Proposition 1.4 (The $\Psi$-Hopf module structure on $R(G)$ ). For the smooth, finite length representation $\pi$ of the group $\mathrm{GL}(m, D)$, and smooth, finite length representation $\sigma$ of the group $G_{k}(D)$ we have

$$
\mu^{*}(\pi \rtimes \sigma)=\Psi^{*}(\pi) \rtimes \mu^{*}(\sigma) .
$$

Proof. As in the split case in [Tadić 1995].

Proposition 1.5 ( $R$-groups). Let $\sigma_{1}, \sigma_{2}, \ldots, \sigma_{k}$ denote discrete series representations of general linear groups over the division algebra $D$, and $\tau$ a discrete series representation of $G_{r}(D)$. The representation

$$
\sigma_{1} \times \sigma_{2} \times \cdots \times \sigma_{k} \rtimes \tau
$$

is multiplicity-free and has length $2^{d}$, where $d$ is the number of mutually nonequivalent $\sigma_{i}$ such that $\sigma_{i} \rtimes \tau$ reduces.

Proof. This is proved in [Hanzer 2004].

Structure and reducibility results for $G_{1}(D)$. It is easy to see that

$$
G_{1}(D)=\left\{\left[\begin{array}{ll}
a d_{1} & b d_{1} \\
c d_{1} & d d_{1}
\end{array}\right] ; a, b, c, d \in F, d_{1} \in D,(a d-b c) d_{1} \tau\left(d_{1}\right)=1\right\} .
$$

So there is an epimorphism

$$
\phi: G_{1}(D) \rightarrow F^{*} /\left(F^{*}\right)^{2}, \phi(g)=(a d-b c)\left(F^{*}\right)^{2}
$$

whose kernel is isomorphic to $\operatorname{SL}(2, F) D_{1}$, where $D_{1}$ denotes the subgroup of elements of norm 1 in $D^{*}$, and is realized as a subgroup of diagonal matrices in $G_{1}(D)$. Also $\operatorname{SL}(2, F) \cap D_{1}=\{ \pm I\}$. 
From now on, for irreducible smooth representations of $D^{*}$ (which are always finite-dimensional) we will use $\chi$ to denote (unitary) characters and $\tau$ to denote (unitary) higher-dimensional representations. The distinction is important, because, by the Jacquet-Langlands correspondence, characters correspond to twists of the Steinberg representation of GL $(2, F)$, and higher-dimensional representations of $D^{*}$ correspond to the cuspidal representations of $\operatorname{GL}(2, F)$ [Bernstein et al. 1984]. Each (continuous) character $\chi$ of $D^{*}$ is of the form $\chi=\chi^{\prime} \circ$ det, for some character $\chi^{\prime}$ of the field $F$.

Proposition 1.6. Assume $\tau$ and $\chi$ are irreducible admissible representations of $D^{*}$.

(i) If $\tau \not \tilde{\tau}$, then $\tau \nu^{s} \rtimes 1$ is irreducible for all $s \in \mathbb{R}$.

(ii) If $\chi^{2} \neq 1$, then $\chi \nu^{s} \rtimes 1$ is irreducible for all $s \in \mathbb{R}$

(iii) Assume $\tau \cong \tilde{\tau}$. Then $\tau v^{s} \rtimes 1$ reduces if and only if $s= \pm \frac{1}{2}$ and $\omega_{\tau}=1$ or $s=0$ and $\omega_{\tau} \neq 1$.

(iv) If $\chi^{2}=1, \chi v^{s} \rtimes 1$ reduces if and only if $s= \pm \frac{1}{2}$.

In both cases, when we have reducibility at $s= \pm \frac{1}{2}$, the induced representation has length 2 and one of the subquotients is a square-integrable representation, denoted by $\delta\left[\chi v^{1 / 2} ; 1\right]$ (or $\left.\delta\left[\tau v^{1 / 2} ; 1\right]\right)$. When the representation $\tau \rtimes 1$ reduces, it is a direct sum of the two nonequivalent tempered representations. The squareintegrable representations obtained this way are mutually inequivalent.

Proof. Let $w_{0}$ be the unique nontrivial element of the Weyl group of $G_{1}(D)$. Applying the standard result of Harish-Chandra [Ban 1999], and taking into account the action of the Weyl group, the representation $\tau \nu^{s} \rtimes 1$ of $G_{1}(D)$ reduces for some $s \in \mathbb{R}$ only if $\tau^{*} \cong \tilde{\tau} \cong \tau$. So we assume that $\tau \cong \tilde{\tau}$. Let

$$
A_{w_{0}}(\tau, s) f_{s}(e)=\int_{U} f_{s}\left(w_{0}^{-1} u\right) d u
$$

be the action of the standard intertwining operator, where $f$ denotes a function in the "compact" picture of the representation $\tau \rtimes 1$ and $f_{s}$ is its analytic section. Make the identification $U \cong F$. We have the explicit calculation:

$$
A_{w_{0}}(\tau, s) f_{s}(e)=\int_{|n| \leq 1} f_{s}\left(w_{0}^{-1} n\right) d n+\sum_{k=1}^{\infty} q^{-2 k s} \omega_{\tau}\left(\bar{\omega}^{k}\right) \int_{O^{*}} f\left(\left[\begin{array}{cc}
u^{-1} & 0 \\
\bar{\omega}^{k} & u
\end{array}\right]\right) d u \text {. }
$$

We denote by $K_{m}$ the $m$-th congruence subgroup in $\operatorname{GL}(2, D)$. If we denote the first integral above, which always converges, by $I_{1}$, and if $f$ is $\left(K_{m} \cap G_{1}(D)\right)$ right-invariant, we get 


$$
\begin{aligned}
A_{w_{0}}(\tau, s) f_{s}(e)=I_{1}+\sum_{k=1}^{m-1} q^{-2 k s} \omega_{\tau}\left(\bar{\omega}^{k}\right) \int_{O^{*}} \omega_{\tau}(u) f\left(\left[\begin{array}{cc}
1 & 0 \\
u^{-1} \bar{\omega}^{k} & 1
\end{array}\right]\right) d u & \\
& +\sum_{k=m}^{\infty} q^{-2 k s} \omega_{\tau}\left(\bar{\omega}^{k}\right) f(e) \int_{O^{*}} \omega_{\tau}(u) d u .
\end{aligned}
$$

We conclude that the operator $A_{w_{0}}(\tau, s)$ is holomorphic at $s=0$ if and only if the central character $\omega_{\tau}$ of the representation $\tau$ is nontrivial. By the results of Harish-Chandra, in this situation the induced representation is reducible at $s=0$, and this is the only point of the reducibility [Silberger 1980].

Now consider the self-contragredient representations $\tau$ (or $\chi$ ) with trivial central character so the induced representation is irreducible at $s=0$.

We determine the poles of the Plancherel measure by computing the composition of the intertwining operators $A_{w_{0}}(\tau, s) A_{w_{0}}(\tau,-s)$. Set $\tilde{f}_{s}=A_{w_{0}}(\tau,-s) f_{-s}$. Then

$$
\begin{aligned}
A_{w_{0}}(\tau, s) \tilde{f}_{s}(e) & =\int_{F}\left|\xi_{0}\right|^{-2 s-1} \tilde{f}_{s}\left(\left[\begin{array}{cc}
1 & 0 \\
\xi_{0}^{-1} & 1
\end{array}\right]\right) d \xi_{0} \\
& =\int_{F}\left|\xi_{0}\right|^{-2 s-1} \int_{F}|\xi|^{2 s-1} f_{-s}\left(\left[\begin{array}{cc}
1 & 0 \\
\xi^{-1}+\xi_{0}^{-1} & 1
\end{array}\right]\right) d \xi d \xi_{0} .
\end{aligned}
$$

To detect the poles of the Plancherel measure, it is enough to consider an $f$ such that $\operatorname{supp} f_{-s} \subset P_{0} \bar{U}_{0}$ and such that

$$
\left.f_{-s}\right|_{\bar{U}_{0}}\left(\left[\begin{array}{ll}
1 & 0 \\
\xi & 1
\end{array}\right]\right)= \begin{cases}0 & \text { if }|\xi|>1 \\
v_{0} & \text { if }|\xi| \leq 1\end{cases}
$$

The vector $v_{0}$ belongs to the representation space of $\tau$. After some simple calculations, we conclude that the composition of those intertwining operators is trivial only for $s= \pm \frac{1}{2}$. (Note that the characters of $D^{*}$ having order at most 2 are necessarily trivial on $F^{*}$.)

\section{The principal series representations}

In this section we write down all the composition factors for the principal series representations, identifying the occurrence of square-integrable and tempered irreducible subquotients.

Recall that to each square-integrable representation of $\operatorname{GL}(n, D)$ is attached a segment of cuspidal representations [Tadić 1990]. So, the (essentially) unique square-integrable subquotient of the representation $\rho v_{\rho}^{k} \times \rho v_{\rho}^{k-1} \times \cdots \times \rho$ is denoted by $\delta\left(\rho v_{\rho}^{k}, \rho\right)$. Here $\rho$ denotes the cuspidal representation of some $\operatorname{GL}(m, D)$. In our case, $v_{\chi}=v^{2}$ for segments of characters of $D^{*}$ and $v_{\tau}=v$ for segments of higher dimensional irreducible cuspidal representations of $D^{*}$. 
The next two propositions describe the composition series of all principal series induced from the characters. Lemma 1.3 and Proposition 1.6 give the reducibility points.

Proposition 2.1. Let $\chi_{1}$ be a unitary character of $D^{*}$ and take $\alpha \in \mathbb{R}$. In the Grothendieck group $R\left(G_{2}(D)\right)$, we have

$$
\begin{aligned}
& \chi_{1} v^{\alpha} \times \chi_{1} v^{\alpha+2} \rtimes 1= \\
& \left\{\begin{array}{lr}
L\left(\chi_{1} v^{3 / 2} \delta\left(v, v^{-1}\right) ; 1\right)+\pi_{1}+L\left(\chi_{1} v^{5 / 2} ; \delta\left[\chi_{1} v^{1 / 2} ; 1\right]\right) & L\left(\chi_{1} v^{5 / 2}, \chi_{1} v^{1 / 2} ; 1\right) \\
L\left(\chi_{1} v^{1 / 2} \delta\left(v, v^{-1}\right) ; 1\right)+\pi_{2}+L\left(\chi_{1} v^{3 / 2} ; \delta\left[\chi_{1} v^{1 / 2} ; 1\right]\right) & \text { if } \chi_{1}^{2}=1, \alpha=\frac{1}{2}, \\
& L\left(\chi_{1} v^{3 / 2}, \chi_{1} v^{1 / 2} ; 1\right) \\
L\left(\chi_{1} v^{\alpha+1} \delta\left(v, v^{-1}\right) ; 1\right)+L\left(\chi_{1} v^{\alpha+2}, \chi_{1} v^{\alpha} ; 1\right) & \text { if } \chi_{1}^{2}=1, \alpha=-\frac{1}{2}, \\
L\left(\chi_{1} v^{1} \delta\left(v, v^{-1}\right) ; 1\right)+L\left(\chi_{1} v^{2} ; \chi_{1} \rtimes 1\right) & \text { if } \alpha \in \mathbb{R}^{+} \backslash\left\{\frac{1}{2}\right\}, \\
L\left(\chi_{1} v^{\alpha+1} \delta\left(v, v^{-1}\right) ; 1\right)+L\left(\chi_{1} v^{\alpha+2}, \chi_{1}^{-1} v^{-\alpha} ; 1\right) & \text { if } \alpha \in(-1,0) \backslash\left\{-\frac{1}{2}\right\}, \\
L\left(\chi_{1} \delta\left(v, v^{-1}\right) \rtimes 1\right)+L\left(\chi_{1} v, \chi_{1}^{-1} v ; 1\right) & \text { if } \alpha=-1, \\
L\left(\chi_{1}^{-1} v^{-1-\alpha} \delta\left(v, v^{-1}\right) ; 1\right)+L\left(\chi_{1}^{-1} v^{-\alpha}, \chi_{1} v^{\alpha+2} ; 1\right) & \text { if } \alpha \in(-2,-1), \\
L\left(\chi_{1}^{-1} v \delta\left(v, v^{-1}\right) ; 1\right)+L\left(\chi_{1}^{-1} v^{2} ; \chi_{1} \rtimes 1\right) & \text { if } \alpha=-2, \\
L\left(\chi_{1}^{-1} v^{-1-\alpha} \delta\left(v, v^{-1}\right) ; 1\right)+L\left(\chi_{1}^{-1} v^{-\alpha}, \chi_{1}^{-1} v^{-\alpha-2} ; 1\right) & \text { if } \alpha<-2 .
\end{array}\right.
\end{aligned}
$$

The representations $\pi_{1}$ and $\pi_{2}$ are square-integrable and mutually inequivalent.

Proof. In the course of the proof we will make extensive use of [Tadic 1998, Remark 3.2 and Lemma 3.7]. We have

$$
\chi_{1} v^{\alpha} \times \chi_{1} v^{\alpha+2} \rtimes 1=\chi_{1} v^{\alpha+1} \delta\left(v, v^{-1}\right) \rtimes 1+L\left(\chi_{1} v^{\alpha+2}, \chi_{1} v^{\alpha}\right) \rtimes 1 .
$$

Using the $\Psi$-Hopf module structure (Proposition 1.4) we obtain

$$
\begin{aligned}
& s_{(2)}\left(\chi_{1} v^{\alpha+1} \delta\left(v, v^{-1}\right) \rtimes 1\right) \\
& \quad \chi_{1} v^{\alpha+1} \delta\left(v, v^{-1}\right) \otimes 1+\chi_{1}^{-1} v^{-(\alpha+1)}\left(\delta\left(v, v^{-1}\right)\right)^{\sim} \otimes 1+\chi_{1}^{-1} v^{-\alpha} \times \chi_{1} v^{\alpha+2} \otimes 1
\end{aligned}
$$

and

$$
s_{(1)}\left(\chi_{1} v^{\alpha+1} \delta\left(v, v^{-1}\right) \rtimes 1\right)=\chi_{1} v^{\alpha+2} \otimes \chi v^{\alpha} \rtimes 1+\chi_{1}^{-1} v^{-\alpha} \otimes \chi v^{\alpha+2} \rtimes 1 .
$$

First, assume that all three expressions $\chi_{1}^{-1} v^{-\alpha} \times \chi_{1} v^{\alpha+2} \otimes 1, \chi v^{\alpha} \rtimes 1$ and $\chi v^{\alpha+2} \rtimes 1$ are irreducible. Then, applying [Tadić 1998, Lemma 3.7] on Jacquet subquotients, we see that, in that case, the representation $\chi_{1} v^{\alpha+1} \delta\left(v, v^{-1}\right) \rtimes 1$ is irreducible. In general (without assumptions on the reducibility of those three expressions), with the aid of the Aubert involution [1995], we conclude that $\chi_{1} v^{\alpha+1} \delta\left(v, v^{-1}\right) \rtimes 1$ and 
$L\left(\chi_{1} v^{\alpha+2}, \chi_{1} v^{\alpha}\right) \rtimes 1$ have the same length. So with the previous assumption, the representation $\chi_{1} v^{\alpha} \times \chi_{1} v^{\alpha+2} \rtimes 1$ has length 2 .

Second, assume $\chi_{1}^{-1} v^{-\alpha} \times \chi_{1} v^{\alpha+2}$ is reducible. This implies $\chi_{1}^{2}=1$ and $\alpha \in$ $\{0,-2\}$. For each choice of $\alpha$ from this set, we get representations which are the same in the Grothendieck group. We have

$$
\chi_{1} v^{2} \times \chi_{1} \rtimes 1=\chi_{1} v \delta\left(v, v^{-1}\right) \rtimes 1+\chi_{1} v 1_{\mathrm{GL}(2, D)} \rtimes 1 .
$$

Lemma 2.2. The representation $\chi_{1} v \delta\left(v, v^{-1}\right) \rtimes 1$ is irreducible.

Proof. We can apply ideas from [Tadić 1998, Section 6].

Third, assume that $\chi v^{\alpha} \rtimes 1$ is reducible. It follows that $\chi_{1}^{2}=1$ and $\alpha \in\left\{ \pm \frac{1}{2}\right\}$. The case $\alpha=\frac{1}{2}$ will be addressed first. We have (in the Grothendieck group)

$$
\begin{aligned}
\chi_{1} v^{5 / 2} \times \chi_{1} v^{1 / 2} \rtimes 1 & =\chi_{1} v^{3 / 2} \delta\left(v, v^{-1}\right) \rtimes 1+\chi_{1} v^{3 / 2} 1_{\mathrm{GL}(2, D)} \rtimes 1 \\
& =\chi_{1} v^{5 / 2} \rtimes \delta\left[\chi_{1} v^{1 / 2} ; 1\right]+\chi_{1} v^{5 / 2} \rtimes L\left(\chi_{1} v^{1 / 2} ; 1\right) .
\end{aligned}
$$

We have

$$
\begin{aligned}
& s_{(2)}\left(\chi_{1} v^{3 / 2} \delta\left(v, v^{-1}\right) \rtimes 1\right)= \\
& \chi_{1} v^{3 / 2} \delta\left(v, v^{-1}\right) \otimes 1+\chi_{1} v^{-3 / 2} \delta\left(v, v^{-1}\right) \otimes 1+\chi_{1} v^{5 / 2} \times \chi_{1} v^{-1 / 2} \otimes 1, \\
& s_{(2)}\left(\chi_{1} v^{5 / 2} \rtimes \delta\left[\chi_{1} v^{1 / 2} ; 1\right]\right)=\chi_{1} v^{1 / 2} \times \chi_{1} v^{5 / 2} \otimes 1+\chi_{1} v^{-5 / 2} \times \chi_{1} v^{1 / 2} \otimes 1 .
\end{aligned}
$$

From this, applying [Tadić 1998, Remark 3.2], it follows that both

$$
\chi_{1} v^{3 / 2} \delta\left(v, v^{-1}\right) \rtimes 1 \text { and } \chi_{1} v^{5 / 2} \rtimes \delta\left[\chi_{1} v^{1 / 2} ; 1\right]
$$

are reducible representations and that they have an irreducible subquotient in common. Examining Jacquet modules in (3) and (4), we conclude that there is only one such subquotient, denoted $\pi_{1}$, and it is a square-integrable representation. Analogously we conclude that $\chi_{1} v^{3 / 2} 1_{\mathrm{GL}(2, D)} \rtimes 1$ and $\chi_{1} v^{5 / 2} \rtimes \delta\left[\chi_{1} v^{1 / 2} ; 1\right]$ have a common irreducible subquotient. We also conclude that each of the representations which appear on the right-hand side of (2) has length at most 3 . If we explore Jacquet modules of the representation $\chi_{1} v^{5 / 2} \rtimes \delta\left[\chi_{1} v^{1 / 2} ; 1\right]$ with respect to the minimal parabolic subgroup, we see that this is impossible. So we obtain the decomposition of the principal series into 4 irreducible subquotients.

In the case $\alpha=-\frac{1}{2}$, the discussion is similar, but here we find a common squareintegrable subquotient $\pi_{2}$ in $\chi_{1} v^{1 / 2} \delta\left(v, v^{-1}\right) \rtimes 1$ and $\chi_{1} v^{3 / 2} \rtimes L\left(\chi_{1} v^{1 / 2} ; 1\right)$. Examining the Jacquet modules with respect to the minimal parabolic subgroup, we find that the principal series has length 4 .

Finally, the reducibility of $\chi_{1} v^{\alpha+2} \rtimes 1$ leads to the representations already seen above. 
Proposition 2.3. We assume $\chi_{1}^{2}=1$. Then

$$
\begin{aligned}
& \chi_{2} v^{\alpha} \times \chi_{1} v^{1 / 2} \rtimes 1= \\
& \left\{\begin{array}{lr}
L\left(\chi_{1} v^{3 / 2} \delta\left(v, v^{-1}\right) ; 1\right)+\pi_{1}+L\left(\chi_{1} v^{5 / 2} ; \delta\left[\chi_{1} v^{1 / 2} ; 1\right]\right)+L\left(\chi_{1} v^{5 / 2}, \chi_{1} v^{1 / 2} ; 1\right) \\
& \text { if } \chi_{2}=\chi_{1}, \alpha=\frac{5}{2}, \\
L\left(\chi_{1} v^{1 / 2} \delta\left(v, v^{-1}\right) ; 1\right)+\pi_{2}+L\left(\chi_{1} v^{3 / 2} ; \delta\left[\chi_{1} v^{1 / 2} ; 1\right]\right)+L\left(\chi_{1} v^{3 / 2}, \chi_{1} v^{1 / 2} ; 1\right) & \text { if } \chi_{2}=\chi_{1}, \alpha=\frac{3}{2}, \\
L\left(\chi_{2} v^{1 / 2} ; \delta\left[\chi_{1} v^{1 / 2} ; 1\right]\right)+\pi_{3}+L\left(\chi_{1} v^{1 / 2}, \chi_{2} v^{1 / 2} ; 1\right)+L\left(\chi_{1} v^{1 / 2} ; \delta\left[\chi_{2} v^{1 / 2} ; 1\right]\right) \\
\chi_{2} \rtimes \delta\left[\chi_{1} v^{1 / 2} ; 1\right]+L\left(\chi_{1} v^{1 / 2} ; \chi_{2} \rtimes 1\right) & \text { if } \chi_{2}^{2}=1, \chi_{2} \neq \chi_{1}, \alpha=\frac{1}{2}, \\
& \text { if } \alpha=0, \\
L\left(\chi_{2} v^{\alpha} ; \delta\left[\chi_{1} v^{1 / 2} ; 1\right]\right)+L\left(\chi_{2} v^{\alpha}, \chi_{1} v^{1 / 2} ; 1\right) & \text { and in other cases: } \\
L\left(\chi_{2}^{-1} v^{-\alpha} ; \delta\left[\chi_{1} v^{1 / 2} ; 1\right]\right)+L\left(\chi_{2}^{-1} v^{-\alpha}, \chi_{1} v^{1 / 2} ; 1\right) & \text { if } \alpha<0<.
\end{array}\right.
\end{aligned}
$$

Moreover, $\pi_{1}, \pi_{2}, \pi_{3}$ are mutually inequivalent, square-integrable representations. Proof. We have $\chi_{2} v^{\alpha} \times \chi_{1} v^{1 / 2} \rtimes 1=\chi_{2} v^{\alpha} \rtimes \delta\left[\chi_{1} v^{1 / 2} ; 1\right]+\chi_{2} v^{\alpha} \rtimes L\left(\chi_{1} v^{1 / 2} ; 1\right)$. Also $s_{(2)}\left(\chi_{2} v^{\alpha} \rtimes \delta\left[\chi_{1} v^{1 / 2} ; 1\right]\right)=\chi_{2} v^{\alpha} \times \chi_{1} v^{1 / 2}+\chi_{2}^{-1} v^{-\alpha} \times \chi_{1} v^{1 / 2}$ and $s_{(1)}\left(\chi_{2} v^{\alpha} \rtimes \delta\left[\chi_{1} v^{1 / 2} ; 1\right]\right)$

$$
=\chi_{1} v^{1 / 2} \otimes \chi_{2} v^{\alpha} \rtimes 1+\chi_{2} v^{\alpha} \otimes \delta\left[\chi_{1} v^{1 / 2} ; 1\right]+\chi_{2}^{-1} v^{-\alpha} \otimes \delta\left[\chi_{1} v^{1 / 2} ; 1\right] .
$$

The assumption that $\chi_{2} v^{\alpha} \times \chi_{1} v^{1 / 2}, \chi_{2}^{-1} v^{-\alpha} \times \chi_{1} v^{1 / 2}$ and $\chi_{2} v^{\alpha} \rtimes 1$ are irreducible, together with [Tadić 1998, Lemma 3.7], lead to the conclusion that $\chi_{2} v^{\alpha} \rtimes$ $\delta\left[\chi_{1} v^{1 / 2} ; 1\right]$ and $\chi_{2} v^{\alpha} \rtimes L\left(\chi_{1} v^{1 / 2} ; 1\right)$ are irreducible. If we drop these assumptions, the only new case to consider is $\chi_{2} v^{1 / 2} \times \chi_{1} v^{1 / 2} \rtimes 1$, with $\chi_{2}^{2}=1$.

First, suppose that $\chi_{2}=\chi_{1}$. The representations $\chi_{1} v^{1 / 2} \rtimes \delta\left[\chi_{1} v^{1 / 2} ; 1\right]$ and $\chi_{1} v^{1 / 2} \rtimes L\left(\chi_{1} v^{1 / 2} ; 1\right)$ are irreducible. Namely, the representation $\chi_{1} v^{1 / 2} \times \chi_{1} v^{-1 / 2}$ is an irreducible unitarizable representation of $\operatorname{GL}(2, D)$, so the representation $\chi_{1} v^{1 / 2} \times \chi_{1} v^{-1 / 2} \rtimes 1$ is also unitarizable and $\chi_{1} v^{1 / 2} \rtimes \delta\left[\chi_{1} v^{1 / 2} ; 1\right]$ is its quotient. But the latter is also a standard representation, so it is irreducible.

Next suppose that $\chi_{2} \neq \chi_{1}$. By examining $s_{(2)}\left(\chi_{2} v^{1 / 2} \rtimes \delta\left[\chi_{1} v^{1 / 2} ; 1\right]\right)$ we see that $\chi_{2} v^{1 / 2} \rtimes \delta\left[\chi_{1} v^{1 / 2} ; 1\right]$ has length at most 2. Also we see from [Tadić 1998, Remark 3.2] that this representation and $\chi_{1} v^{1 / 2} \rtimes L\left(\chi_{2} v^{1 / 2} ; 1\right)$ have one common subquotient, a square-integrable representation denoted $\pi_{3}$.

Now, we describe the composition factors of all principal series induced from higher-dimensional representations. The principal series representation of the form $\tau_{1} v^{\alpha} \times \tau_{2} v^{\beta} \rtimes 1$ where $\tau_{1}, \tau_{2}$ have dimension greater than 1 , are reducible only in the situations covered by the next four propositions. 
Proposition 2.4. Let $\tau_{1}$ denote an irreducible, admissible, unitary representation of $D^{*}$ of dimension greater than 1 . If $\tau_{1}$ is not a selfdual representation, we have

$$
\begin{aligned}
& \tau_{1} v^{\alpha+1} \times \tau_{1} v^{\alpha} \rtimes 1= \\
& \qquad \begin{cases}L\left(v^{\alpha+\frac{1}{2}} \delta\left(\tau_{1} v^{1 / 2}, \tau_{1} v^{-1 / 2}\right) ; 1\right)+L\left(\tau_{1} v^{\alpha+1}, \tau_{1} v^{\alpha} ; 1\right) & \text { if } \alpha>0, \\
L\left(v^{1 / 2} \delta\left(\tau_{1} v^{1 / 2}, \tau_{1} v^{-1 / 2}\right) ; 1\right)+L\left(\tau_{1} v ; \tau_{1} \rtimes 1\right) & \text { if } \alpha=0, \\
L\left(v^{\alpha+\frac{1}{2}} \delta\left(\tau_{1} v^{1 / 2}, \tau_{1} v^{-1 / 2}\right) ; 1\right)+L\left(\tau_{1} v^{\alpha+1}, \tilde{\tau}_{1} v^{-\alpha} ; 1\right) & \text { if } \alpha \in\left(-\frac{1}{2}, 0\right), \\
\delta\left(\tau_{1} v^{1 / 2}, \tau_{1} v^{-1 / 2}\right) \rtimes 1+L\left(\tau_{1} v^{1 / 2}, \tilde{\tau}_{1} v^{-1 / 2} ; 1\right) & \text { if } \alpha=-\frac{1}{2}, \\
L\left(v^{-\alpha-\frac{1}{2}} \delta\left(\tilde{\tau}_{1} v^{1 / 2}, \tilde{\tau}_{1} v^{-1 / 2}\right) ; 1\right)+L\left(\tilde{\tau}_{1} v^{-\alpha}, \tau_{1} v^{\alpha+1} ; 1\right) & \text { if } \alpha \in\left(-1,-\frac{1}{2}\right), \\
L\left(v^{-\alpha-\frac{1}{2}} \delta\left(\tilde{\tau}_{1} v^{1 / 2}, \tilde{\tau}_{1} v^{-1 / 2}\right) ; 1\right)+L\left(\tilde{\tau}_{1} v^{-\alpha}, \tilde{\tau}_{1}^{-\alpha-1} ; 1\right) & \text { if } \alpha<-1 .\end{cases}
\end{aligned}
$$

Proof. We have

$$
\left.\tau_{1} v^{\alpha+1} \times \tau_{1} v \rtimes 1=v^{1 / 2} \delta\left(\tau_{1} v^{1 / 2}, \tau_{1} v^{-1 / 2}\right)\right) \rtimes 1+v^{1 / 2} L\left(\tau_{1} v^{1 / 2}, \tau_{1} v^{-1 / 2}\right) \rtimes 1 .
$$

Analogously to the proof of the previous proposition, we examine

$$
\begin{aligned}
\left.s_{(2)}\left(v^{1 / 2+\alpha} \delta\left(\tau_{1} v^{1 / 2}, \tau_{1} v^{-1 / 2}\right)\right) \rtimes 1\right)=v^{1 / 2+\alpha} \delta\left(\tau_{1} v^{1 / 2}, \tau_{1} v^{-1 / 2}\right) \otimes 1 \\
+v^{-1 / 2-\alpha}\left(\delta\left(\tau_{1} v^{1 / 2}, \tau_{1} v^{-1 / 2}\right)\right)^{\sim} \otimes 1+\tilde{\tau}_{1} v^{-\alpha} \times \tau_{1} v^{\alpha+1} \otimes 1 .
\end{aligned}
$$

Also $s_{(1)}\left(v^{1 / 2+\alpha} \delta\left(\tau_{1} v^{1 / 2}, \tau_{1} v^{-1 / 2}\right)\right)=\tau_{1} v^{\alpha+1} \otimes \tau_{1} v^{\alpha} \rtimes 1+\tilde{\tau}_{1} v^{-\alpha} \otimes \tau_{1} v^{\alpha+1} \rtimes 1$. With the assumptions that $\tilde{\tau}_{1} v^{-\alpha} \times \tau_{1} v^{\alpha+1}, \tau_{1} v^{\alpha} \rtimes 1$, and $\tau_{1} v^{\alpha+1} \rtimes 1$ are irreducible, and applying [Tadić 1998, Lemma 3.7], we obtain that the representation $\left(v^{1 / 2+\alpha} \delta\left(\tau_{1} v^{1 / 2}, \tau_{1} v^{-1 / 2}\right) \rtimes 1\right.$ is irreducible. By the properties of the Aubert involution, also that the representation $v^{1 / 2} L\left(\tau_{1} v^{1 / 2}, \tau_{1} v^{-1 / 2}\right) \rtimes 1$ is irreducible. These assumptions are met when $\tau_{1} \nsucceq \tilde{\tau}_{1}$.

Proposition 2.5. Let $\tau_{1}$ denote an irreducible, unitary, selfdual representation of $D^{*}$ of dimension greater than 1 . Without loss of generality we can assume $\alpha \geq-\frac{1}{2}$.

(i) If $\chi_{\tau_{1}}=1$ we have

$$
\begin{aligned}
& \tau_{1} v^{\alpha+1} \times \tau_{1} v^{\alpha} \rtimes 1= \\
& \left\{\begin{array}{lc}
L\left(v^{1 / 2} \delta\left(\tau_{1} v^{1 / 2}, \tau_{1} v^{-1 / 2}\right) ; 1\right)+L\left(\tau_{1} v ; \tau_{1} \rtimes 1\right) & \text { if } \alpha=0, \\
L\left(v \delta\left(\tau_{1} v^{1 / 2}, \tau_{1} v^{-1 / 2}\right) ; 1\right)+L\left(\tau_{1} v^{3 / 2} ; \delta\left[\tau v^{1 / 2} ; 1\right]\right)+\pi_{4}+L\left(\tau_{1} v^{3 / 2}, \tau_{1} v^{1 / 2} ; 1\right) \\
L\left(\tau_{1} v^{1 / 2} ; \delta\left[\tau_{1} v^{1 / 2} ; 1\right]\right)+L\left(\tau v_{1}^{1 / 2}, \tau v_{1}^{1 / 2} ; 1\right)+T_{1}+T_{2} & \text { if } \alpha=\frac{1}{2}, \\
L\left(v^{\alpha+\frac{1}{2}} \delta\left(\tau_{1} v^{1 / 2}, \tau_{1} v^{-1 / 2}\right) ; 1\right)+L\left(\tau_{1} v^{\alpha+1}, \tau_{1} v^{-\alpha} ; 1\right) & \text { if } \alpha=-\frac{1}{2}, \\
L\left(v^{\alpha+\frac{1}{2}} \delta\left(\tau_{1} v^{1 / 2}, \tau_{1} v^{-1 / 2}\right) ; 1\right)+L\left(\tau_{1} v^{\alpha+1}, \tau_{1} v^{\alpha} ; 1\right) & \text { if } \alpha \in\left(-\frac{1}{2}, 0\right),
\end{array}\right.
\end{aligned}
$$


(ii) If $\chi_{\tau_{1}} \neq 1$ and $\tau_{1} \rtimes 1=T_{3}^{\prime}+T_{4}^{\prime}$ we have

$$
\begin{aligned}
& \tau_{1} v^{\alpha+1} \times \tau_{1} v^{\alpha} \rtimes 1= \\
& \begin{cases}L\left(\tau_{1} v ; T_{3}^{\prime}\right)+L\left(\tau_{1} v ; T_{4}^{\prime}\right)+2 L\left(v^{1 / 2} \delta\left(\tau_{1} v^{1 / 2}, \tau_{1} v^{-1 / 2}\right) ; 1\right)+\pi_{5}+\pi_{6} & \text { if } \alpha=0, \\
L\left(v^{\alpha+\frac{1}{2}} \delta\left(\tau_{1} v^{1 / 2}, \tau_{1} v^{-1 / 2}\right) ; 1\right)+L\left(\tau_{1} v^{\alpha+1}, \tau_{1} v^{\alpha} ; 1\right) & \text { if } \alpha>0, \\
\delta\left(\tau_{1} v^{1 / 2}, \tau_{1} v^{-1 / 2}\right) \rtimes 1+L\left(\tau_{1} v^{1 / 2}, \tau_{1} v^{1 / 2} ; 1\right) & \text { if } \alpha=-\frac{1}{2}, \\
L\left(v^{\alpha+\frac{1}{2}} \delta\left(\tau_{1} v^{1 / 2}, \tau_{1} v^{-1 / 2}\right) ; 1\right)+L\left(\tau_{1} v^{\alpha+1}, \tau_{1} v^{-\alpha} ; 1\right) & \text { if } \alpha \in\left(-\frac{1}{2}, 0\right) .\end{cases}
\end{aligned}
$$

Moreover, $\pi_{4}, \pi_{5}$ and $\pi_{6}$ are mutually inequivalent square-integrable representations, and $T_{1}, T_{2}$ and $\delta\left(\tau_{1} v^{1 / 2}, \tau_{1} v^{-1 / 2}\right) \rtimes 1$ in the second case are mutually inequivalent tempered (not square-integrable) representations.

Proof. Dropping the assumptions that $\tilde{\tau}_{1} v^{-\alpha} \times \tau_{1} v^{\alpha+1}, \tau_{1} v^{\alpha} \rtimes 1$, and $\tau_{1} v^{\alpha+1} \rtimes 1$ are irreducible (see proof of the previous proposition), we are left to deal with the following families of representations in (5)-(8) below:

$$
\tau_{1} v \times \tau_{1} \rtimes 1 \text {, when } \chi_{\tau_{1}}=1 \text {. }
$$

Analogously to Lemma 2.2 we conclude that $v^{1 / 2} \delta\left(\tau_{1} v^{1 / 2}, \tau_{1} v^{-1 / 2}\right) \rtimes 1$ is irreducible. Another representation to consider is

$$
\tau_{1} v \times \tau_{1} \rtimes 1 \text {, when } \chi_{\tau_{1}} \neq 1 \text {. }
$$

Here we obtain a single case where multiplicity one fails; this is also the only induced representation of length 6 . Examining the Jacquet modules we learn that the representation $\tau_{1} \rtimes T_{3}^{\prime}$ has length at most 3 and that it is reducible (because it has the same length as $\tau_{1} \rtimes T_{4}^{\prime}$ ). If we assume that it has length 2 , then also

$$
v^{1 / 2} \delta\left(\tau_{1} v^{1 / 2}, \tau_{1} v^{-1 / 2}\right) \rtimes 1=L\left(v^{1 / 2} \delta\left(\tau_{1} v^{1 / 2}, \tau_{1} v^{-1 / 2}\right) ; 1\right)+\pi,\left(\text { in } R\left(G_{2}(D)\right)\right),
$$

where $\pi$ is some subrepresentation. We see that then $L\left(v^{1 / 2} \delta\left(\tau_{1} v^{1 / 2}, \tau_{1} v^{-1 / 2}\right) ; 1\right)$ has to be a subrepresentation of $\tau_{1} v \times \tau_{1} \rtimes 1$, but $v^{1 / 2} \delta\left(\tau_{1} v^{1 / 2}, \tau_{1} v^{-1 / 2}\right) \rtimes 1$ is also a subrepresentation of $\tau_{1} v \times \tau_{1} \rtimes 1$. This leads to conclusion that either $L\left(v^{1 / 2} \delta\left(\tau_{1} v^{1 / 2}, \tau_{1} v^{-1 / 2}\right) ; 1\right)$ is a subrepresentation of $v^{1 / 2} \delta\left(\tau_{1} v^{1 / 2}, \tau_{1} v^{-1 / 2}\right) \rtimes 1$, or the multiplicity of $L\left(v^{1 / 2} \delta\left(\tau_{1} v^{1 / 2}, \tau_{1} v^{-1 / 2}\right)\right.$; 1$)$ in $\tau_{1} v \times \tau_{1} \rtimes 1$ is greater then 1 ; both of them false (in this situation). So we conclude that $\tau_{1} \rtimes T_{3}^{\prime}$ and $\tau_{1} \rtimes T_{4}^{\prime}$ both have length 3 , and both have unique subrepresentations which are square-integrable (denoted $\pi_{5}$ and $\pi_{6}$ ). By careful examination of the composition sequences of the Jacquet modules, we conclude that the representations $v^{1 / 2} \delta\left(\tau_{1} v^{1 / 2}, \tau_{1} v^{-1 / 2}\right) \rtimes 1$ and $v^{1 / 2} L\left(\tau_{1} v^{1 / 2}, \tau_{1} v^{-1 / 2}\right) \rtimes 1$ have one irreducible quotient in common. In the 
Grothendieck group we have

$$
\begin{aligned}
v^{1 / 2} \delta\left(\tau_{1} v^{1 / 2}, \tau_{1} v^{-1 / 2}\right) \rtimes 1 & =L\left(v^{1 / 2} \delta\left(\tau_{1} v^{1 / 2}, \tau_{1} v^{-1 / 2}\right) ; 1\right)+\pi_{5}+\pi_{6}, \\
\tau_{1} v \rtimes T_{3}^{\prime} & =L\left(\tau_{1} v ; T_{3}^{\prime}\right)+\pi_{5}+L\left(v^{1 / 2} \delta\left(\tau_{1} v^{1 / 2}, \tau_{1} v^{-1 / 2}\right) ; 1\right) .
\end{aligned}
$$

The next case is

$$
\tau_{1} v^{3 / 2} \times \tau_{1} v^{1 / 2} \rtimes 1 \quad \text { if } \chi_{\tau_{1}}=1 .
$$

Examining the length of Jacquet modules, similarly to the case of inducing from the characters, we see that the length of $\nu \delta\left(\tau_{1} v^{1 / 2}, \tau_{1} v^{-1 / 2}\right) \rtimes 1$ can't be 3 , because that's inconsistent with the associativity of Jacquet modules. The rest is straightforward. We use a similar analysis to deal with

$$
\tau_{1} v^{1 / 2} \times \tau_{1} v^{1 / 2} \rtimes 1 \quad \text { if } \chi_{\tau_{1}}=1 .
$$

Proposition 2.6. Let $\tau_{2}$ be a unitary, irreducible selfdual representation of $D^{*}$ of dimension greater than 1 , with trivial central character, and let $\tau_{1}$ denote a unitary irreducible representation of $D^{*}$ of dimension greater than 1 .

(a) If $\tau_{1} ¥ \tilde{\tau}_{1}$, we have

$$
\tau_{1} v^{\alpha} \times \tau_{2} v^{1 / 2} \rtimes 1= \begin{cases}L\left(\tau_{1} v^{\alpha} ; \delta\left[\tau_{2} v^{1 / 2} ; 1\right]\right)+L\left(\tau_{1} v^{\alpha}, \tau_{2} v^{1 / 2} ; 1\right) & \text { if } \alpha>0, \\ T_{3}+L\left(\tau_{2} v^{1 / 2} ; \tau_{1} \rtimes 1\right) & \text { if } \alpha=0,\end{cases}
$$

where $T_{3}=\tau_{1} \rtimes \delta\left[\tau_{2} v^{1 / 2} ; 1\right]$ is an irreducible tempered representation.

(b) If $\tau_{1} \cong \tilde{\tau}_{1}$, we consider two cases:

(i) If $\chi_{\tau_{1}}=1$, then $\tau_{1} v^{\alpha+1} \times \tau_{1} v^{\alpha} \rtimes 1=$

$$
\left\{\begin{array}{lr}
L\left(\tau_{1} v^{\alpha} ; \delta\left[\tau_{2} v^{1 / 2} ; 1\right]\right)+L\left(\tau_{1} v^{\alpha}, \tau_{2} v^{1 / 2} ; 1\right) & \text { if }|\alpha| \notin \mathbb{R}_{0}^{+} \backslash\left\{0, \frac{1}{2}, \frac{3}{2}\right\}, \\
L\left(\tau_{1} v^{1 / 2} ; \delta\left[\tau_{2} v^{1 / 2} ; 1\right]\right)+L\left(\tau_{2} v^{1 / 2} ; \delta\left[\tau_{1} v^{1 / 2} ; 1\right]\right)+L\left(\tau_{1} v^{1 / 2}, \tau_{2} v^{1 / 2} ; 1\right)+\pi_{7} \\
L\left(\tau_{1} v^{1 / 2} ; \delta\left[\tau_{1} v^{1 / 2} ; 1\right]\right)+L\left(\tau v_{1}^{1 / 2}, \tau v_{1}^{1 / 2} ; 1\right)+T_{1}+T_{2} & \text { if }|\alpha|=\frac{1}{2} \text { and } \tau_{1} \cong \tau_{2}, \\
\tau_{1} \times \delta\left[\tau_{2} v^{1 / 2} ; 1\right]+L\left(\tau_{2} v^{1 / 2} ; \tau_{1} \rtimes 1\right) & \text { if } \alpha=0, \\
L\left(v \delta\left(\tau_{1} v^{1 / 2}, \tau_{1} v^{-1 / 2}\right) ; 1\right)+L\left(\tau_{1} v^{3 / 2} ; \delta\left[\tau v^{1 / 2} ; 1\right]\right)+\pi_{4}+L\left(\tau_{1} v^{3 / 2}, \tau_{1} v^{1 / 2} ; 1\right) & \text { if }|\alpha|=\frac{3}{2} \text { and } \tau_{1} \cong \tau_{2}, \\
L\left(\tau_{1} v^{3 / 2} ; \delta\left[\tau_{2} v^{1 / 2} ; 1\right]\right)+L\left(\tau_{1} v^{3 / 2}, \tau_{2} v^{1 / 2} ; 1\right) & \text { if }|\alpha|=\frac{3}{2} \text { and } \tau_{1} \nsucceq \tau_{2} .
\end{array}\right.
$$

The representation $\pi_{7}$ is square-integrable, and $\tau_{1} \times \delta\left[\tau_{2} v^{1 / 2} ; 1\right]$ is an irreducible tempered representation. 
(ii) If $\chi_{\tau_{1}} \neq 1$ and $\tau_{1} \rtimes 1=T_{3}^{\prime}+T_{4}^{\prime}$, then

$$
\tau_{1} v^{\alpha} \times \tau_{2} v^{1 / 2} \rtimes 1= \begin{cases}L\left(\tau_{1} v^{\alpha} ; \delta\left[\tau_{2} v^{1 / 2} ; 1\right]\right)+L\left(\tau_{1} v^{\alpha}, \tau_{2} v^{1 / 2} ; 1\right) & \text { if } \alpha>0, \\ L\left(\tau_{2} v^{1 / 2} ; T_{3}^{\prime}\right)+L\left(\tau_{2} v^{1 / 2} ; T_{4}^{\prime}\right)+T_{5}+T_{4} & \text { if } \alpha=0 .\end{cases}
$$

$T_{4}$ and $T_{5}$ are irreducible tempered representations.

Proof. The only new case left to check, after dealing with ones which are covered by [Tadić 1998, Lemma 3.7], is

$$
\tau_{1} v^{1 / 2} \times \tau_{2} v^{1 / 2} \rtimes 1 \quad \text { if } \tau_{1} ¥ \tau_{2}, \chi_{\tau_{1}}=\chi_{\tau_{2}}=1 .
$$

This case is resolved in the same way as for the characters.

Proposition 2.7. Let $\tau_{2}$ be a unitary, irreducible, self-dual representation of $D^{*}$ such that $\chi_{\tau_{2}} \neq 1$, so that $\tau_{2} \rtimes 1=T_{3}^{\prime} \oplus T_{4}^{\prime}$, and let $\tau_{1}$ be an irreducible unitary representation of $D^{*}$.

(a) If $\alpha>0$, then $\tau_{1} v^{\alpha} \times \tau_{2} \rtimes 1=$

$$
\left\{\begin{array}{lc}
L\left(\tau_{1} v^{1 / 2} ; T_{3}^{\prime}\right)+L\left(\tau_{1} v^{1 / 2} ; T_{4}^{\prime}\right)+T_{4}+T_{5} & \text { if } \alpha=\frac{1}{2}, \tau_{1} \cong \tilde{\tau}_{1}, \omega_{\tau_{1}}=1, \\
L\left(\tau_{2} v ; T_{3}^{\prime}\right)+L\left(\tau_{2} v ; T_{4}^{\prime}\right)+2 L\left(v^{1 / 2} \delta\left(\tau_{2} v^{1 / 2}, \tau_{2} v^{-1 / 2}\right) ; 1\right)+\pi_{5}+\pi_{6} \\
& \text { if } \tau_{1} \cong \tau_{2} \text { and } \alpha=1, \\
L\left(\tau_{1} v^{\alpha} ; T_{3}^{\prime}\right)+L\left(\tau_{1} v^{\alpha} ; T_{4}^{\prime}\right) & \text { in other cases } .
\end{array}\right.
$$

(b) If $\alpha=0$, then

$$
\tau_{1} \times \tau_{2} \rtimes 1= \begin{cases}T_{6}+T_{7}+T_{8}+T_{9} & \text { if } \tau_{1} \cong \tilde{\tau}_{1}, \omega_{\tau_{1}} \neq 1, \tau_{1} ¥ \tau_{2}, \\ T_{10}+T_{11} & \text { in other cases. }\end{cases}
$$

The representations $T_{i}, i=6, \ldots, 11$, are mutually inequivalent, tempered (not square-integrable) representations.

Proof. The cases in the first part of this Proposition were already covered, and the statements of the second part follow from [Hanzer 2004].

We now settle the mixed case of the principal series representations.

Proposition 2.8. Let $\chi$ be a unitary character of $D^{*}$, and let $\tau$ be irreducible, admissible unitary representation of $D^{*}$ of dimension greater than 1 . Then the principal series representation $\chi v^{\alpha} \times \tau \nu^{\beta} \rtimes 1$ (for $\left.\alpha, \beta \in \mathbb{R}\right)$ reduces only in the following cases: 
(i) If $\chi^{2}=1$, then $\tau v^{\beta} \times \chi^{1 / 2} \rtimes 1=$

$$
\begin{cases}L\left(\tau v^{\beta} ; \delta\left[\chi v^{1 / 2} ; 1\right]\right)+L\left(\tau v^{\beta}, \chi v^{1 / 2} ; 1\right) & \text { if } \beta>0 \text { and } \tau v^{\beta} \rtimes 1 \text { is irreducible, } \\ \tau \rtimes \delta\left[\chi v^{1 / 2} ; 1\right]+L\left(\chi v^{1 / 2} ; \tau \rtimes 1\right) & \text { if } \beta=0 \text { and } \tau \rtimes 1 \text { is irreducible, } \\ T_{7}+T_{8}+L\left(\chi v^{1 / 2}, T_{3}^{\prime}\right)+L\left(\chi v^{1 / 2}, T_{4}^{\prime}\right) & \text { if } \tau \cong \tilde{\tau}, \omega_{\tau} \neq 1, \beta=0, \\ L\left(\tau v^{1 / 2} ; \delta\left[\chi v^{1 / 2} ; 1\right]\right)+\pi_{8}+L\left(\chi v^{1 / 2} ; \delta\left[\tau v^{1 / 2} ; 1\right]\right)+L\left(\tau v^{1 / 2}, \chi v^{1 / 2} ; 1\right) & \text { if } \tau \cong \tilde{\tau}, \omega_{\tau}=1,|\beta|=\frac{1}{2},\end{cases}
$$

where, in the third case, $\tau \rtimes 1=T_{3}^{\prime}+T_{4}^{\prime}$. The tempered representation $\tau \rtimes$ $\delta\left[\chi v^{1 / 2} ; 1\right]$ from the second case is irreducible, and the representation $\pi_{8}$ is square-integrable.

(ii) If $\tau v^{1 / 2} \rtimes 1$ reduces, then $\chi v^{\alpha} \times \tau v^{1 / 2} \rtimes 1=$

$$
\begin{cases}L\left(\chi v^{\alpha} ; \delta\left[\tau v^{1 / 2} ; 1\right]\right)+L\left(\chi v^{\alpha}, \tau v^{1 / 2} ; 1\right) & \text { if } \alpha>0 \text { and } \chi v^{\alpha} \rtimes 1 \text { is irreducible, } \\ L\left(\tau v^{1 / 2} ; \delta\left[\chi v^{1 / 2} ; 1\right]\right)+L\left(\chi v^{1 / 2} ; \delta\left[\tau v^{1 / 2} ; 1\right]\right)+L\left(\tau v^{1 / 2}, \chi v^{1 / 2} ; 1\right)+\pi & \\ \chi \rtimes \delta\left[\tau v^{1 / 2} ; 1\right]+L\left(\tau v^{1 / 2} ; \chi \rtimes 1\right) & \text { if } \chi^{2}=1 \text { and } \alpha=\frac{1}{2}, \\ & \text { if } \alpha=0 .\end{cases}
$$

The tempered representation $\chi \rtimes \delta\left[\tau \nu^{1 / 2} ; 1\right]$ in the third case is irreducible.

(iii) If $\tau \rtimes 1=T_{3}^{\prime} \oplus T_{4}^{\prime}$, then $\chi v^{\alpha} \times \tau \rtimes 1=$

$$
\begin{cases}L\left(\chi v^{\alpha} ; T_{3}^{\prime}\right)+L\left(\chi v^{\alpha} ; T_{4}^{\prime}\right) & \text { if } \alpha>0 \text { and } \chi v^{\alpha} \rtimes 1 \text { is irreducible, } \\ \chi \rtimes T_{3}^{\prime}+\chi \rtimes T_{4}^{\prime} & \text { if } \alpha=0, \\ T_{7}+T_{8}+L\left(\chi v^{1 / 2}, T_{3}^{\prime}\right)+L\left(\chi v^{1 / 2}, T_{4}^{\prime}\right) & \text { if } \chi^{2}=1,|\alpha|=\frac{1}{2} .\end{cases}
$$

Proof. Use [Tadić 1998, Lemma 3.7].

\section{Induced representations of the group $G_{2}(D)$; the Siegel case}

We now consider the reducibility of the representations of the form

$$
\sigma v^{s} \rtimes 1
$$

where $\sigma$ is an irreducible admissible cuspidal representation of $\operatorname{GL}(2, D)$ and $s$ is a real number. By a result of Harish-Chandra [Ban 1999], if this induced representation is reducible for some $s, \sigma$ must be self-contragredient. So, from now on, we assume that $\sigma \cong \tilde{\sigma}$. Let $\sigma^{\prime}$ be the square-integrable representation of $\operatorname{GL}(4, F)$ corresponding to $\sigma$ by the Jacquet-Langlands correspondence. It is actually a cuspidal representation as well [Bernstein et al. 1984].

Proposition 3.1. Let $\sigma$ be an irreducible, admissible, selfdual cuspidal representation of the group $\operatorname{GL}(2, D)$. The representation $\sigma \rtimes 1$ is irreducible if and only if 
$L\left(s, \sigma^{\prime}, \Lambda^{2} \rho_{4}\right)$ has a pole at $s=0$. If this is so, the representation $\sigma v^{s} \rtimes 1$, where $s \in \mathbb{R}$, reduces only for $s= \pm \frac{1}{2}$.

Proof. Recall that the Plancherel measure is defined as

$$
R(s, \sigma, \bar{N}(F), N(F)) R(s, \sigma, N(F), \bar{N}(F))=\mu^{-1}(s, \sigma) .
$$

Our notation is as in [Muić and Savin 2000]. From that paper we know that

$$
\mu(s, \sigma)=\mu\left(s, \sigma^{\prime}\right),
$$

where on the left-hand side we have the Plancherel measure in the group $G_{2}(D)$, and on the right-hand side the Plancherel measure corresponding to the representation induced from $\sigma^{\prime}$ in $\mathrm{SO}(8, F)$. Because $\sigma^{\prime}$ is cuspidal, the reducibility of $\sigma^{\prime} v^{s} \rtimes 1$ can be obtained directly from the Plancherel measure: there exists a unique $s_{0} \geq 0$ such that $\sigma^{\prime} \nu^{s_{0}} \rtimes 1$ reduces [Silberger 1980] and

$$
\begin{array}{ll}
s_{0}=0 & \text { if and only if } \mu\left(0, \sigma^{\prime}\right) \neq 0, \\
s_{0}>0 & \text { if and only if } \mu\left(s, \sigma^{\prime}\right) \text { has a pole at } s=s_{0} .
\end{array}
$$

So, $\sigma v^{s} \rtimes 1$ is reducible if and only if $\sigma^{\prime} v^{s} \rtimes 1$ is reducible, and $s_{0} \in\left\{\frac{1}{2}, 0\right\}$, by the results in [Shahidi 1990b]. Because $\sigma^{\prime}$ is generic, the Plancherel measure is expressible in terms of $L$-functions. To conclude, $\sigma^{\prime} \rtimes 1$ is irreducible if and only if $L\left(s, \sigma^{\prime}, \Lambda^{2} \rho_{4}\right)$ has a pole at $s=0$; see [Shahidi 1992].

\section{Induced representations of the group $G_{2}(D)$; the non-Siegel case}

We now consider the representations of the form

$$
\tau \nu^{s} \rtimes \delta,
$$

where $\tau$ is an irreducible admissible unitary representation of $D^{*}, \delta$ is an irreducible cuspidal representation of $G_{1}(D)$, and $s \in \mathbb{R}$. As in the previous section, to examine the reducibility, it is enough to assume that $\tau \cong \tilde{\tau}$, and $s \geq 0$. Throughout this section we keep this assumption. For an algebraic number field $k$, we denote its ring of adeles by $A_{k}$. We consider the restriction

$$
\left.\delta\right|_{\mathrm{SL}(2, F) D_{1}}=\sum_{i=1}^{k} \tau_{i} \otimes \delta_{i},
$$

according to the observation about the structure of $G_{1}(D)$ - see Equation (1). The procedure we use is this: we choose a summand in the restriction above, such as $\tau_{1} \otimes \delta_{1}$, and lift it to the discrete series representation $\tau_{1} \otimes \delta_{1}^{\prime}$ of the group $\operatorname{SL}(2, F) \times \operatorname{SL}(2, F)$. Then we find representations $\delta^{\prime}$ and $\delta^{\prime \prime}$ of $\mathrm{SO}(4, F)$ such that the representation $\tau_{1} \otimes \delta_{1}^{\prime}$ is a component in the restrictions of the representations 
$\delta^{\prime}$ and $\delta^{\prime \prime}$ to $\operatorname{SL}(2, F) \cdot \operatorname{SL}(2, F)$. Then, using global methods, we will prove that $\mu(s, \tau \otimes \delta)^{2}=\mu\left(s, \tau^{\prime} \otimes \delta^{\prime}\right) \mu\left(s, \tau^{\prime} \otimes \delta^{\prime \prime}\right)$. The difficulty in applying the global methods lies in that there are global $L$-packets for the group $\operatorname{SL}\left(2, A_{k}\right)$ including both automorphic and nonautomorphic global representations [Labesse and Langlands 1979], so we have to make some adjustments. Also, in order to ensure that the representations $\delta^{\prime}$ and $\delta^{\prime \prime}$ differ only in quadratic character, i.e., they have the same restriction to $\operatorname{SL}(2, F) \cdot \operatorname{SL}(2, F)$, we have to be careful when varying representations in the local $L$-packets of $\tau_{1}$ and $\delta_{1}$. Before we proceed with the detailed exposition, briefly remind the reader how the group $\operatorname{SL}(2, F) \cdot \operatorname{SL}(2, F)$ sits in $\mathrm{SO}(4, F)$. Let $\{\alpha, \beta\}$ denote the basis of the root system $\Phi(\mathrm{SO}(4, F), T)$, where $T$ is a diagonal subgroup in the standard matrix realization of $\operatorname{SO}(4, F)$. So, with the obvious meaning, we choose $\alpha=e_{1}-e_{2}$ and $\beta=e_{1}+e_{2}$. The Levi subgroup $M_{\alpha}$ corresponding to the root $\alpha$ is isomorphic to $\operatorname{GL}(2, F)$; the same is true for $M_{\beta}$. One copy of SL $(2, F)$ is standardly embedded in $M_{\alpha}$ and the other in $M_{\beta}$; one is block-diagonal, and the other is not.

We can choose a number field $k$ having two places $v_{1}$ and $v_{2}$ such that $k_{v_{1}} \cong$ $k_{v_{2}} \cong F$, and a division algebra $\mathbf{D}$ of rank 4 over $k$ that it splits only at $v_{1}$ and $v_{2}$, with $\mathbf{D}_{v_{1}} \cong D \cong \mathbf{D}_{v_{1}}$. Then we can define the reductive group $\mathbf{G}_{1}$ over $k$ such that $\mathbf{G}_{1}\left(k_{v}\right) \cong \mathrm{SO}\left(4, k_{v}\right)$ for all $v \notin\left\{v_{1}, v_{2}\right\}$ and $\mathbf{G}_{1}\left(k_{v_{1}}\right) \cong G_{1}(D) \cong \mathbf{G}_{1}\left(k_{v_{2}}\right)$. Analogously, we define $\mathbf{G}_{2}$ over $k$. Also, we can define $\mathbf{D}_{1}$, the subgroup of elements of norm 1 in $\mathbf{D}^{*}$ such that $\mathbf{D}_{1}\left(k_{v}\right) \cong \operatorname{SL}\left(2, k_{v}\right)$ for $v \notin\left\{v_{1}, v_{2}\right\}$ and $\mathbf{D}_{1}\left(k_{v_{1}}\right) \cong D_{1} \cong \mathbf{D}_{1}\left(k_{v_{2}}\right)$. We choose any of the summands from the restriction of $\delta$, e.g., $\tau_{1} \otimes \delta_{1}$.

First assume that $\operatorname{dim} \delta_{1}>1$. Consider the set of ideles $\left(( \pm I)_{v}\right)$ that can be observed as a subgroup of $\mathbf{D}_{1}$. We can form the character $\omega=\prod \omega_{v}$ on that set such that $\omega_{v_{1}}=\omega_{\delta_{1}}, \omega_{v_{2}}=\omega_{\delta_{1}}$, and $\omega_{v}$ are almost everywhere trivial. Then we can introduce the space $L\left(\mathbf{D}_{1}\left(A_{k}\right)\right.$ ) (and other notation) as in [Flicker 1987], and study the representations of the functions from $C\left(\mathbf{D}_{1}\left(A_{k}\right)\right)$ on the space $L\left(\mathbf{D}_{1}\left(A_{k}\right)\right)$. We choose a full tensor $f=\otimes f_{v}$ from the space $C\left(\mathbf{D}_{1}\left(A_{k}\right)\right)$. We can choose $f$ in such a way that $f_{v_{1}}$ and $f_{v_{2}}$ are the coefficients of the representation $\delta_{1}$, and at all other nonarchimedean places $f_{v}$ are spherical. Then we can adjust the support of $f_{v}$ at the archimedean places in such way that we can reason analogously to [Flicker 1987, Proposition $\$ 3.3$ and Theorem §4.3]. We obtain the existence of an automorphic cuspidal representation $\pi_{1}^{\prime}=\bigotimes_{v} \pi_{1, v}^{\prime}$ of the group $\mathbf{D}_{1}\left(A_{k}\right)$ with central character $\omega$ such that $\pi_{1, v_{1}}^{\prime} \cong \delta_{1} \cong \pi_{1, v_{2}}^{\prime}$ Then there exists a grossencharacter $\omega^{\prime}=\bigotimes_{v} \omega_{v}^{\prime}$ such that $\left.\omega^{\prime}\right|_{\left(\left( \pm I_{v}\right)\right)}=\omega$. Also, we can find an automorphic cuspidal representation $\pi^{\prime}=\bigotimes_{v} \pi_{v}^{\prime}$ of the group $\mathbf{D}^{*}\left(A_{k}\right)$ with central character $\omega^{\prime}$ such that $\pi_{1}^{\prime}$ embeds in $\pi^{\prime}$; the proof is analogous to one in [Flicker 1992]. Note that $\pi_{v_{1}}^{\prime}$ and $\pi_{v_{2}}$ are cuspidal representations of $D^{*}$ of dimension greater then 1 , so by the Jacquet-Langlands correspondence, they correspond to cuspidal representations of 
GL(2,F). This enables us to use results of [Flicker and Kazhdan 1988] about lifts of representations of $\mathbf{D}^{*}\left(A_{k}\right)$ to the representations of $\mathrm{GL}\left(2, A_{k}\right)$ with one fixed cuspidal place. So there exists an automorphic cuspidal representation $\pi=\bigotimes_{v} \pi_{v}$ of GL(2, $\left.A_{k}\right)$ such that $\pi_{v} \cong \pi_{v}^{\prime}$ for all $v \notin\left\{v_{1}, v_{2}\right\}$, and $\pi_{v_{i}}$ and $\pi_{v_{i}}^{\prime}$ correspond. Let $\pi_{1}=\bigotimes_{v} \pi_{1, v}$ denote some automorphic cuspidal representation of $\operatorname{SL}\left(2, A_{k}\right)$ embedded in the representation $\pi \mid \operatorname{SL}\left(2, A_{k}\right)$. We can arrange that $\pi_{1, v} \cong \pi_{1, v}^{\prime}$ for every place $v$ different from $v_{1}, v_{2}$. Indeed, let $\{\phi\}$ be an admissible homomorphism $\{\phi\}: W_{K / k} \rightarrow P G L(2) \times W_{K / k}$ defined by the representation $\operatorname{Ind}\left(W_{K / k}, W_{K / E}, \theta\right)$, where $K$ is some large, but finite Galois extension of $k, E$ a quadratic extension of $k$ contained in $K$, and $\theta$ a Grossencharacter of $E$ that doesn't factor through $N m_{E / k}$. Let $\pi_{2}$ be some automorphic cuspidal representation of $\operatorname{SL}\left(2, A_{k}\right)$ embedded in $\pi$. If $\pi_{2}$ does not belong to the $L$-packet parameterized by $\{\phi\}$, we define a representation $\pi_{1}$ of $\operatorname{SL}\left(2, A_{k}\right)$ in the following way:

$$
\begin{aligned}
\pi_{1, v}=\pi_{1, v}^{\prime} & \text { for all } v \notin\left\{v_{1}, v_{2}\right\}, \\
\pi_{1, v_{i}}=\pi_{2, v_{i}} & \text { for } i=1,2 .
\end{aligned}
$$

The representation $\pi_{1}$ is in the same $L$-packet as $\pi_{2}$ and it is automorphic; see [Labesse and Langlands 1979]. If $\pi_{2}$ corresponds to $\{\phi\}$ as above, we can form $\pi_{1}$ as above at split places, but at $v_{1}$ and $v_{2}$ we must adjust representations to obtain a representation which is in the same $L$-packet as $\pi_{2}$ but is also automorphic. We can do so because the multiplicity with which $\pi_{1}$ occurs in the space of cusp forms is

$$
\frac{1}{\left[S_{\phi}^{\circ} \backslash S_{\phi}\right]} \sum_{s \in S_{\phi}^{\circ} S_{\phi}}\left\langle s, \pi_{1}\right\rangle,
$$

with notation as in [Labesse and Langlands 1979]. So we want to make

$$
\left\langle s, \pi_{1}\right\rangle=\prod\left\langle s, \pi_{1, v}\right\rangle
$$

a trivial character. But we can easily do that fixing at the place $v_{1}$ the representation which defines the trivial character on the local group $S_{\phi_{v_{1}}}^{\circ} \backslash S_{\phi_{v_{1}}}$, and adjusting accordingly at the place $v_{2}$.

Second, if $\operatorname{dim} \delta_{1}=1$, i.e., $\delta_{1}=1$, we fix a nonarchimedean place $u$ outside $\left\{v_{1}, v_{2}\right\}$ and fix some cuspidal representation $\pi_{u}$ of $\operatorname{SL}\left(2, k_{u}\right)$ at that place. As before, we can choose an automorphic cuspidal representation $\pi_{1}^{\prime}$ of $\mathbf{D}_{1}$ which has that component on the place $u$, and which is unramified at the places $v_{1}$ and $v_{2}$, i.e., equal to $\delta_{1}=1$. Now there exists a lift from the automorphic cuspidal representations of $\mathbf{D}^{*}$ to such representations of $\operatorname{GL}\left(2, A_{k}\right)$, with fixed place $u$ with cuspidal component, and, as before, we obtain the representation of $\operatorname{SL}\left(2, A_{k}\right)$ having properties as in the previous case.

If the finite set $\left\{g_{i}=\left[\begin{array}{ll}1 & 0 \\ 0 & x_{i}\end{array}\right]\right\}$ is a set of representatives of $\operatorname{GL}(2, F) / \operatorname{SL}(2, F) F^{*}$, 
then the set

$$
g_{i}^{\prime}=\left\{\left[\begin{array}{cccc}
1 & 0 & 0 & 0 \\
0 & x_{i} & 0 & 0 \\
0 & 0 & x_{i}^{-1} & 0 \\
0 & 0 & 0 & 1
\end{array}\right]\right\}
$$

is a set of representatives (in our realization) of $\mathrm{SO}(4, F) /(\mathrm{SL}(2, F) \cdot \mathrm{SL}(2, F))$. Now, with the same character $\omega$ as before, we can find an automorphic cuspidal representation $\varepsilon=\bigotimes_{v} \varepsilon_{v}$ of $\operatorname{SL}\left(2, A_{k}\right)$ with the central character $\omega$ such that

$$
\begin{array}{ll}
\varepsilon_{v_{1}} \cong \varepsilon_{v_{2}} \cong \tau_{1} & \text { if } \pi_{1, v_{1}} \cong \pi_{1, v_{2}}, \\
\varepsilon_{v_{1}} \cong \tau_{1}, \varepsilon_{v_{2}} \cong{ }^{g_{i}} \tau_{1} & \text { if } \pi_{1, v_{2}} \cong{ }^{g_{i}} \pi_{1, v_{1}} .
\end{array}
$$

There exists an automorphic cuspidal representation $\sigma^{\prime}$ of the group $\mathbf{G}_{1}\left(A_{k}\right)$ in which $\varepsilon \otimes \pi_{1}^{\prime}$ embeds as a representation of the group $\operatorname{SL}\left(2, A_{k}\right) \mathbf{D}_{1}\left(A_{k}\right)$. Analogously, there exists an automorphic cuspidal representation $\sigma$ of $\operatorname{SO}\left(4, A_{k}\right)$ in which $\varepsilon \otimes \pi_{1}$ embeds as a representation of $\operatorname{SL}\left(2, A_{k}\right) \cdot \operatorname{SL}\left(2, A_{k}\right)$. We can arrange that

$$
\sigma_{v_{1}}^{\prime} \cong \sigma_{v_{2}}^{\prime} \cong \delta \quad \text { and } \quad \sigma_{v}^{\prime} \cong \sigma_{v} \quad \text { for all } v \notin\left\{v_{1}, v_{2}\right\} .
$$

Let $\gamma^{\prime}=\bigotimes_{v} \gamma_{v}^{\prime}$ be an automorphic cuspidal representation of $\mathbf{D}^{*}\left(A_{k}\right)$ such that $\gamma_{v_{1}}^{\prime} \cong \gamma_{v_{2}}^{\prime} \cong \tau$ and let $\gamma$ be its lift to GL(2; $\left.A_{k}\right)$ such that $\gamma_{v}^{\prime} \cong \gamma_{v}$ for all $v \notin$ $\left\{v_{1}, v_{2}\right\}$ and $\gamma_{v_{1}} \cong \gamma_{v_{2}} \cong \tau^{\prime}$, where $\tau^{\prime}$ corresponds to $\tau$ by Jacquet-Langlands correspondence. This can be arranged; see [Flicker and Kazhdan 1988].

We have to normalize measures on the unipotent radicals of the groups considered in order to get the global functional equation right. We can decompose $D$ as $F \oplus D^{-}$, looking at the center $F$ of the algebra $D$ as the $\tau$-hermitian part of $D$ and $D^{-}$as the $\tau$-antihermitian part. Now, the unipotent radical of the non-Siegel parabolic subgroup in the group $G_{2}(D)$ in the case $\varepsilon=-1$ (the case we are now considering) is $N(F) \cong D \oplus D \oplus F$, and in the case $\varepsilon=1$ it is $N^{\prime}(F) \cong D \oplus D \oplus D^{-}$. Let $\psi_{F}$ denote a nontrivial additive character of $F$. Introduce self-dual measures on $N(F)$ and $N^{\prime}(F)$ by the use of the $F$-form $\langle x, y\rangle=\sum_{i=1}^{5} x_{i} y_{i}+\tau\left(x_{i} y_{i}\right)$ on $D^{5}$, and a character $\psi_{F}$ so that the self-dual measure on $D^{5}$ is the product of a self-dual measure $\alpha_{F}$ on $N(F)$ and $\alpha_{F}^{\prime}$ on $N^{\prime}(F)$. Fix a nontrivial character $\psi=\bigotimes_{v} \psi_{v}$ of $A_{k}$ trivial on $k$ and such that $\psi_{v_{1}}=\psi_{v_{2}}=\psi_{F}$. As above, at each split place we can get a self-dual measure $\alpha_{v}$ on $N\left(k_{v}\right)$ with respect to $\psi_{v}$, and a self-dual measure $\alpha$ on $N\left(A_{k}\right)$. In this way, we get a coherent family of measures $\left\{\alpha_{v}\right\}$ such that $\alpha=\prod \alpha_{v}$ and $\alpha$ is actually the Tamagawa measure [Weil 1973, §VII.2, Corollary 1], meaning that $\alpha\left(N\left(A_{k}\right) / N(k)\right)=1$. The Plancherel measure is defined analogously to the Siegel case.

Proposition 4.1. $\quad \mu(s, \tau \otimes \delta)^{2}=\mu\left(s, \tau^{\prime} \otimes \sigma_{v_{1}}\right) \mu\left(s, \tau^{\prime} \otimes \sigma_{v_{2}}\right)$. 
Proof. Denote by $S$ a finite set of places containing $v_{1}$ and $v_{2}$, all the places of residual characteristic 2, and all the places where $\gamma_{v}, \sigma_{v}$, and $\psi_{v}$ are ramified. For every $v \notin S$, let $f_{v, s}$ denote the unique unramified vector in $\gamma_{v}^{\prime} \nu^{s} \rtimes \sigma_{v}^{\prime}$, normalized to be equal to 1 on the maximal compact subgroup $K_{v}$. Analogously, define $\bar{f}_{v, s}$ in $\gamma_{v}^{\prime} v^{s} \rtimes \sigma_{v}^{\prime}$. In the $L$-group $\operatorname{SO}(8, \mathbb{C})$ of $\operatorname{SO}\left(8, k_{v}\right)$, the action (representation) of the $\operatorname{GL}(2, \mathbb{C}) \times \operatorname{SO}(4, \mathbb{C})$ on the unipotent radical is equal to $\Lambda^{2}\left(\mathbb{C}^{2}\right) \oplus\left(\mathbb{C}^{2} \otimes \mathbb{C}^{4}\right)$. So, for $v \notin S$ we can explicitly calculate the constants $c\left(v, s, \gamma_{v}^{\prime} \otimes \sigma_{v}^{\prime}\right)$ in terms of $L$-functions, where

$$
R\left(s, \gamma_{v}^{\prime} \otimes \sigma_{v}^{\prime}, N\left(k_{v}\right), \bar{N}\left(k_{v}\right)\right) f_{v, s}=c\left(v, s, \gamma_{v}^{\prime} \otimes \sigma_{v}^{\prime}\right) \bar{f}_{v, s} .
$$

We then have

$$
c\left(v, s, \gamma_{v}^{\prime} \otimes \sigma_{v}^{\prime}\right)=\frac{L\left(s, \gamma_{v}^{\prime} \otimes \sigma_{v}^{\prime}, \rho_{2} \otimes \rho_{4}\right)\left(1-\chi_{\gamma_{v}^{\prime}}(\bar{\omega}) q_{v}^{-s}\right)^{-1}}{\left.L\left(1+s, \gamma_{v}^{\prime} \otimes \sigma_{v}^{\prime}, \rho_{2} \otimes \rho_{4}\right)\right)\left(1-\chi_{\gamma_{v}^{\prime}}(\bar{\omega}) q_{v}^{-s-1}\right)^{-1}} .
$$

It is easily seen that the product

$$
c_{S}\left(s, \gamma^{\prime} \otimes \sigma^{\prime}\right)=\prod_{v \notin S} c\left(v, s, \gamma_{v}^{\prime} \otimes \sigma_{v}^{\prime}\right)
$$

converges in some right half-plane, and it continues to a meromorphic function on C. Analogously we have

$$
R\left(s, \gamma_{v}^{\prime} \otimes \sigma_{v}^{\prime}, \bar{N}\left(k_{v}\right), N\left(k_{v}\right)\right) \bar{f}_{v, s}=c\left(v,-s, \tilde{\gamma}_{v}^{\prime} \otimes \sigma_{v}^{\prime}\right) f_{v, s} .
$$

We now take $f_{s}=\bigotimes_{v} f_{v, s} \in \gamma^{\prime} v^{s} \rtimes \sigma^{\prime}$, where for each $v \notin S$ we have chosen spherical $f_{v, s}$ as above. Because we have chosen the Tamagawa measure on the (global) unipotent radical we have the global functional equation

$$
R\left(s, \gamma^{\prime} \otimes \sigma^{\prime}, \bar{N}\left(A_{k}\right), N\left(A_{k}\right)\right) R\left(s, \gamma^{\prime} \otimes \sigma^{\prime}, N\left(A_{k}\right), \bar{N}\left(A_{k}\right)\right) f_{s}=f_{s} ;
$$

see [Mœglin and Waldspurger 1995, Theorem IV.1.10]. When the right-hand side of this equation is written as a product of local intertwining operators, the local Plancherel measures appear. So we have

$$
\prod_{v \in S} \mu\left(s, \gamma_{v}^{\prime} \otimes \sigma_{v}^{\prime}\right) c_{S}\left(s, \gamma^{\prime} \otimes \sigma^{\prime}\right) c_{S}\left(-s, \tilde{\gamma}^{\prime} \otimes \sigma^{\prime}\right)=1 .
$$

By analogy with the previous equation for $\mathbf{G}_{2}\left(A_{k}\right)$, we have the equation

$$
\prod_{v \in S} \mu\left(s, \gamma_{v} \otimes \sigma_{v}\right) c_{S}(s, \gamma \otimes \sigma) c_{S}(-s, \tilde{\gamma} \otimes \sigma)=1
$$

in $\mathrm{SO}\left(8, A_{k}\right)$. But at each split place we have an isomorphism $\mathbf{G}_{2}\left(k_{v}\right) \cong \mathrm{SO}\left(8, k_{v}\right)$ that preserves unipotent radicals, and we have isomorphic representations, so we 
have equality of the Plancherel measures. From this, it follows that

$$
\mu(s, \tau \otimes \delta)^{2}=\mu\left(s, \tau^{\prime} \otimes \sigma_{v_{1}}\right) \mu\left(s, \tau^{\prime} \otimes \sigma_{v_{2}}\right) .
$$

Remark. Because of our adjustment of the representation $\varepsilon$, we conclude that the representations $\sigma_{v_{1}}$ and $\sigma_{v_{2}}$ differ in the quadratic character that is trivial on $\operatorname{SL}(2, F) \cdot \operatorname{SL}(2, F)$.

We now compute the Plancherel measure above. Because $\tau_{1}$ is a generic representation for some nontrivial character of $F$ and $\pi_{v_{1}}$ is a generic representation for some nontrivial character, we conclude that $\sigma_{v_{1}}$ is a generic representation of the group $\mathrm{SO}(4, F)$. We can now use [Shahidi 1990b] and express the Plancherel measure in terms of $\gamma$-factors. We fix a nontrivial additive character $\psi$ of $F$ and obtain, up to the exponential factor,

$$
\mu\left(s, \tau^{\prime} \otimes \sigma_{v_{1}}\right) \approx \frac{\gamma\left(2 s, \tau^{\prime}, \Lambda^{2} \rho_{2}, \psi\right) \gamma\left(s, \tau^{\prime} \times \sigma_{v_{1}}\right)}{\gamma\left(1+2 s, \tau^{\prime}, \Lambda^{2} \rho_{2}, \psi\right) \gamma\left(1+s, \tau^{\prime} \times \sigma_{v_{1}}\right)} .
$$

The only difficulty appears in the calculation of the Rankin-Selberg $\gamma$-factor of the groups $\mathrm{GL}(2, F) \times \mathrm{SO}(4, F)$. If $\sigma_{v_{1}}$ is noncuspidal, the computation is straightforward, using the multiplicativity of $\gamma$-factors [Shahidi 1990a]. So, assume that the representations $\sigma_{v_{i}}$ appearing in the previous proposition are square-integrable, noncuspidal representations. This is the case when, with the previous notations, $\delta_{1}=1$. Let $\pi$ denote the cuspidal unitary representation of $\operatorname{GL}(2, F)$ with trivial central character such that $\left.\tau_{1} \hookrightarrow \pi\right|_{\mathrm{SL}(2, F)}$. Then $\pi$ is a self-contragredient representation. Such $\pi$ 's differ mutually by a quadratic character. We denote the basis of the root system for $\mathrm{SO}(4, F)$ by $\{\alpha, \beta\}$. Now, the standard Levi subgroup $M_{\alpha}$ is diagonally embedded in $\mathrm{SO}(4, F)$ and contains the diagonal version of $\operatorname{SL}(2, F)$, and $M_{\beta}$ isn't diagonal and also contains the other copy of $\operatorname{SL}(2, F)$. Consider the representation $\operatorname{Ind}_{M_{\beta}}^{\mathrm{SO}(4, F)} \pi v^{1 / 2}$. It is easy to see that this representation restricted to $\operatorname{SL}(2, F) \cdot \operatorname{SL}(2, F)$ decomposes as

$$
\left.\operatorname{Ind}_{M_{\beta}}^{\mathrm{SO}(4, F)} \pi v^{1 / 2}\right|_{\mathrm{SL}(2, F) \mathrm{SL}(2, F)}=\sum v \rtimes 1 \otimes \tau_{i},
$$

where the $\tau_{i}$ are components of the restriction of $\pi$ to $\operatorname{SL}(2, F)$. Let $\delta^{\prime}$ denote the unique square-integrable subquotient of $\operatorname{Ind}_{M_{\beta}}^{\mathrm{SO}(4, F)} \pi v^{1 / 2}$. Then $\operatorname{St}_{\mathrm{SL}(2, F)} \otimes \tau_{1}$ injects in $\delta^{\prime}$, so $\sigma_{v_{1}}$ and $\sigma_{v_{2}}$ differ from $\delta^{\prime}$ by a quadratic character. We can conclude:

Corollary 4.2. Assume that the representations $\sigma_{v_{i}}$ are not cuspidal and that $\sigma_{v_{1}}$ injects in $\operatorname{Ind}_{P_{\beta}}^{\mathrm{SO}(4, F)} \pi v^{1 / 2}$.

(i) If $\operatorname{dim} \tau>1$ then

(a) if $\chi_{\tau^{\prime}}(\bar{\omega}) \neq 1$ the representation $\tau \nu^{s} \rtimes \delta$ reduces only for $s=0$, and

(b) if $\chi_{\tau^{\prime}}(\bar{\omega})=1$ then $\tau \rtimes \delta$ is irreducible, and $\tau \nu^{s} \rtimes \delta$ reduces at $s=\frac{1}{2}$ or at $s=\frac{3}{2}$, depending on whether $\tau^{\prime} ¥ \pi$ or $\tau^{\prime} \cong \pi$, respectively. 
(ii) If $\tau=\chi$ is a quadratic character, the representation $\chi \nu^{s} \rtimes \delta$ reduces only at $s=\frac{1}{2}$.

Proof. In the formula for Plancherel measure we include the expression for the Rankin-Selberg factor

$$
\gamma\left(s, \tau^{\prime} \times \sigma_{v_{1}}, \psi\right)=\gamma\left(s, \tau^{\prime} \times \pi v^{1 / 2}, \psi\right) \gamma\left(s, \tau^{\prime} \times \pi v^{-1 / 2}, \psi\right) .
$$

If $\tau^{\prime}$ is cuspidal, we obtain the claim, and if $\tau^{\prime}=\chi \operatorname{St}_{\mathrm{GL}(2, F)}$ we have $\gamma\left(s, \chi \operatorname{St}_{\mathrm{GL}(2, F)} \times \pi v^{\frac{1}{2}}, \psi\right)=\gamma\left(s, \chi v^{1 / 2} \times \pi v^{1 / 2}, \psi\right) \gamma\left(s, \chi v^{-1 / 2} \times \pi v^{1 / 2}, \psi\right)=1$.

We denote by ${ }^{\varepsilon}$ the conjugation in $\mathrm{SO}(4, F)$ by the element

$$
\varepsilon=\left[\begin{array}{llll}
0 & 0 & 0 & 1 \\
0 & 1 & 0 & 0 \\
0 & 0 & 1 & 0 \\
1 & 0 & 0 & 0
\end{array}\right]
$$

of $O(4, F) \backslash \mathrm{SO}(4, F)$, and, accordingly, if $\pi$ is the representation of $\mathrm{SO}(4, F)$ we denote the representation of $\mathrm{SO}(4, F)$ obtained using the involution ${ }^{\varepsilon}$ by ${ }^{\varepsilon} \pi$. Keeping the assumptions from the proposition, in the case of cuspidal $\sigma_{v_{i}}$ 's we have

Corollary 4.3. (i) Suppose $\tau=\chi$ is a quadratic character of $D^{*}$.

(a) If ${ }^{\varepsilon} \sigma_{v_{1}} ¥ \sigma_{v_{1}}$ the representation $\chi v^{s} \rtimes \delta$ reduces only at $s=\frac{1}{2}$.

(b) If ${ }^{\varepsilon} \sigma_{v_{1}} \cong \sigma_{v_{1}}$ (so that also ${ }^{\varepsilon} \sigma_{v_{2}} \cong \sigma_{v_{2}}$ ) then if at least one of the representations $\chi \rtimes \tilde{\sigma}_{v_{i}}, i=1,2$, of the group $\mathrm{SO}(6, F)$ is irreducible, $\chi v^{s} \rtimes \delta$ reduces (only) for $s=\frac{3}{2}$. On the other hand, if both of the representations $\chi \rtimes \tilde{\sigma}_{v_{i}}, i=1,2$, reduce, $\chi \nu^{s} \rtimes \delta$ reduces for $s=\frac{1}{2}$.

(ii) Suppose $\operatorname{dim} \tau>1$.

(a) If $\chi_{\tau}(\bar{\omega})=1$ the representation $\tau \nu^{1 / 2} \rtimes \delta$ reduces.

(b) If $\chi_{\tau}(\bar{\omega})=-1$ then the points of reducibility are at $s=0$ or $s=1$. We have reducibility at $s=1$ if at least one of the representations $\tau^{\prime} \rtimes \sigma_{v_{i}}$ of the group $\mathrm{SO}(8, F)$ is irreducible.

Proof. We prove (i). Using the multiplicativity of $\gamma$-factors, we come to $\gamma$-factors $\gamma\left(s, \chi v^{1 / 2} \times \sigma_{v_{i}}\right)$, which leads us to consider the reducibility of the representations $\chi v^{s} \rtimes \sigma_{v_{i}}$ of the group $\operatorname{SO}(6, F)$. It is well known that in order to have reducibility for some real number $s$, the nontrivial element of the Weyl group has to fix the representation $\chi \otimes \sigma_{v_{i}}$, which amounts to the statement that ${ }^{\varepsilon} \sigma_{v_{1}} \cong \sigma_{v_{1}}$. If it isn't so, the aforementioned $L$-functions and $\gamma$-factors appearing in the case of $\operatorname{SO}(6, F)$ 
are trivial, so the only pole of the Plancherel measure comes from the Hecke $L$ function for $\tau^{\prime}$. If ${ }^{\varepsilon} \sigma_{v_{1}} \cong \sigma_{v_{1}}$, the irreducibility of $\chi \rtimes \tilde{\sigma}_{v_{1}}$ implies that $L\left(s, \chi \times \sigma_{v_{1}}\right)$ has a pole at $s=0$. This implies that $\mu(s, \tau \otimes \delta)$ has a pole at $s=\frac{3}{2}$, no matter the situation with $\chi \rtimes \tilde{\sigma}_{v_{2}}$. On the other hand, if both of the representations $\chi \rtimes \tilde{\sigma}_{v_{i}}$ reduce, the Rankin-Selberg $L$-functions are holomorphic for real $s$ and the only poles of $\mu(s, \tau \otimes \delta)$ come from the Hecke $L$-function for $\chi \mathrm{St}_{\mathrm{GL}(2, F)}$.

The proof of (ii) follows from [Goldberg and Shahidi 2001, Theorem 4.8].

\section{Unitary dual of the group $G_{2}(D)$}

We are interested in finding the hermitian, and especially irreducible unitarizable representations of $G_{2}(D)$. We will list them by grouping together the ones with the same cuspidal support.

5.1. Unitary subquotients of the principal series. Let $\chi_{1}$ and $\chi_{2}$ denote unitary characters of $D^{*}$. Let $\pi=\chi_{1} v^{s_{1}} \times \chi_{2} v^{s_{2}} \rtimes 1$. Without loss of generality we can assume that $s_{1} \geq s_{2} \geq 0$.

Proposition 5.1. Assume that we have unitary characters $\chi_{1}$ and $\chi_{2}$ such that $\chi_{1}^{2} \neq 1$ and $\chi_{2}^{2} \neq 1$.

(i) If $\chi_{1} \neq \chi_{2}^{ \pm 1}$ then the representation $\pi$ has a hermitian subquotient if and only if $s_{1}=s_{2}=0$ and then it is an irreducible tempered representation.

(ii) If $\chi_{1}=\chi_{2}$ then the representation $\pi$ has a hermitian subquotient if and only if $s_{1}=s_{2}=0$ and then it is an irreducible tempered representation.

(iii) Suppose if $\chi_{1}=\chi_{2}^{-1}$. If $s_{2}=0$ the representation $\pi$ has a hermitian subquotient only if $s_{1}=0$ and we obtain an irreducible tempered representation (isomorphic to one obtained in the previous case for the same $\chi_{1}$ ).

If $s_{2}>0$ the representation $\pi$ has a hermitian subquotient only if $s_{1}=s_{2}$; then for all $s_{1}>0$ all the subquotients of the representation $\pi$ are hermitian. For $s_{1} \in(0,1)$ we have $\pi=\chi_{1} v^{s_{1}} \times \chi_{1}^{-1} v^{s_{1}} \rtimes 1=L\left(\chi_{1} v^{s_{1}}, \chi_{1}^{-1} v^{s_{1}} ; 1\right)$ and $\pi$ is a unitary representation. For $s_{1}>1, \pi$ is not a unitary representation. For $s_{1}=1$ we have

$$
\pi=\chi_{1} \delta\left(v, v^{-1}\right) \rtimes 1+L\left(\chi_{1} v, \chi_{1}^{-1} v ; 1\right),
$$

where the first subquotient is a tempered representation, and the other is unitary (nontempered).

Proof. The first two cases follow from the criterion for the hermiticity of the Langlands quotient. For the third case we observe that $\chi_{1} v^{s_{1}} \times \chi_{1} v^{-s_{1}}$ is the complementary series of the group $\operatorname{GL}(2, D)$ for $\alpha \in(0,1)$. From this, it follows that $\chi_{1} v^{s_{1}} \times \chi_{1} v^{-s_{1}} \rtimes 1$ has exclusively unitarizable subquotients for $s_{1} \in(0,1]$. 
Proposition 5.2. Let $\chi_{1}$ and $\chi_{2}$ be unitary characters such that $\chi_{1}^{2}=1$ and $\chi_{2}^{2} \neq 1$. Again, let $\pi=\chi_{1} v^{s_{1}} \times \chi_{2} v^{s_{2}} \rtimes 1$. The representation $\pi$ has a hermitian subquotient only if $s_{2}=0$; then all of its subquotients are hermitian representations. In this case, for $s_{1}=0, \pi$ is an irreducible tempered representation; for $s_{1}=\frac{1}{2}$, the representation $\pi$ has an irreducible tempered subquotient; for $s_{1} \in\left(0, \frac{1}{2}\right]$, the representation $L\left(\chi_{1} v^{s_{1}} ; \chi_{2} \rtimes 1\right)$ is unitarizable; and for $s_{1}>\frac{1}{2}$, the representation $\pi$ is irreducible and nonunitarizable.

Proof. We just comment on the case $s_{2}=0$. We have the standard intertwining operators $A_{w_{2 \alpha+\beta}}\left(s_{1}\right): \chi_{1} v^{s_{1}} \times \chi_{2} \rtimes 1 \rightarrow \chi_{1} v^{-s_{1}} \times \chi_{2} \rtimes 1$, which converge for $s_{1}>0$. These operators define, for $s_{1}>\frac{1}{2}$, a continuous family, indexed by $s_{1}$, of nondegenerate hermitian forms on the compact picture $X$ of the representation $\chi_{1} \times \chi_{2} \rtimes 1$ by means of

$$
\left(f_{1}, f_{2}\right)_{s_{1}}=\int_{K}\left\langle f_{1, s_{1}}(k), A_{w_{2 \alpha+\beta}}\left(s_{1}\right) f_{2, s_{1}}(k)\right\rangle d k .
$$

Here $f_{1}$ and $f_{2}$ belong to $X$ and $f_{1, s_{1}}, f_{2, s_{1}}$ denote their holomorphic sections, which are identified with elements from $\chi_{1} v^{s_{1}} \times \chi_{2} \rtimes 1$. If one of these forms were unitarizable, meaning that the irreducible representation $\chi_{1} v^{s_{1}} \times \chi_{2} \rtimes 1$ is unitarizable, all the other forms would have to be unitarizable, too, because of the connectedness of the indexing set. But for $s_{1}>\frac{5}{2}$ the representation $\chi_{1} v^{s_{1}} \times \chi_{2} \rtimes 1$ has unbounded matrix coefficients, which implies nonunitarizability. The operator $A_{w_{2 \alpha+\beta}}\left(s_{1}\right)$ has a pole at $s_{1}=0$, but we can normalize it by multiplying it by an appropriate real polynomial; we obtain, for $s_{1} \in\left[0, \frac{1}{2}\right.$ ), a family of (normalized) intertwining operators $A_{w_{2 \alpha+\beta}}^{\prime}\left(s_{1}\right)$ which also define a continuous family of nondegenerate hermitian forms on $X$. By the same argument, we obtain the unitarizability of the representations considered for $s_{1} \in\left[0, \frac{1}{2}\right)$. In this way, we obtain the complementary series representations, and, by the results in [Miličić 1973], the subquotients of the representation on the edge of the complementary series $\left(s_{1}=\frac{1}{2}\right)$ are unitarizable.

Proposition 5.3. With notation as before, assume that $\chi_{1}^{2} \neq 1$ and $\chi_{2}^{2}=1$. Then the representation $\pi$ has a hermitian subquotient only if $s_{1}=s_{2}=0$, and $\pi$ is then irreducible and tempered. This representation is already described in the previous proposition.

The proof is left to the reader.

Proposition 5.4. Assume that we have unitary characters $\chi_{1}$ and $\chi_{2}$ such that $\chi_{1}^{2}=\chi_{2}^{2}=1$ and $\chi_{1} \neq \chi_{2}$. Let $\pi=\chi_{1} v^{s_{1}} \times \chi_{2} v^{s_{2}} \rtimes 1$. Consider the regions defined on the $s_{1} s_{2}$ plane in Figure 1 (which also includes points that do not have $s_{1} \geq s_{2}$ ).

(i) The representation $\pi$ for $\left(s_{1}, s_{2}\right)$ from the closed region I has all its subquotients unitarizable. The composition factors are given in Proposition 2.3. 


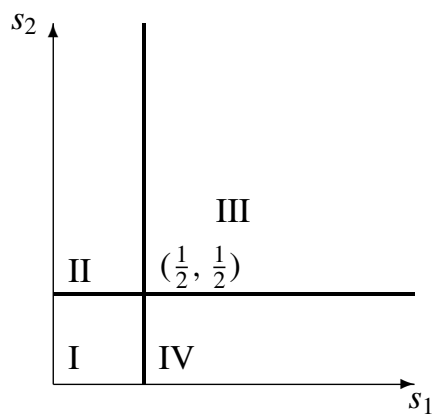

Figure 1

(ii) For $\left(s_{1}, s_{2}\right)$ in each of the open regions II, III, IV we obtain representations all of whose subquotients are hermitian, but none unitarizable.

Proof. For $\left(s_{1}, s_{2}\right)$ with $s_{1} \geq s_{2}>0$ from open region I we have the family of standard long intertwining operators $A\left(s_{1}, s_{2}\right): \chi_{1} v^{s_{1}} \times \chi_{2} v^{s_{2}} \rtimes 1 \rightarrow \chi_{1} v^{-s_{1}} \times$ $\chi_{2} v^{-s_{2}} \rtimes 1$ which defines a continuous family of nondegenerate hermitian forms on the compact picture. So, we can fix such a pair $\left(s_{1}, s_{2}\right)$ and form the one-parameter family $t \rightarrow\left(t s_{1}, t s_{2}\right)$ for $t \geq 0$. Because $\chi_{1} \times \chi_{2} \rtimes 1$ is irreducible, we can assume that we have unitarizable representations for $t \geq 0$, until $\chi_{1} v^{t s_{1}} \times \chi_{2} v^{t s_{2}} \rtimes 1$ starts being reducible. An analogous reasoning and the unboundedness of the matrix coefficients ensures the nonunitarizability of the representations on the unbounded regions. We, of course, could apply immediately [Tadić 1983] to conclude that.

Proposition 5.5. Given a quadratic character $\chi_{1}$ of $D^{*}$, set $\pi=\chi_{1} v^{s_{1}} \times \chi_{1} v^{s_{2}} \rtimes 1$. All subquotients of $\pi$ are hermitian representations. Let the notation be as in Figure 2.

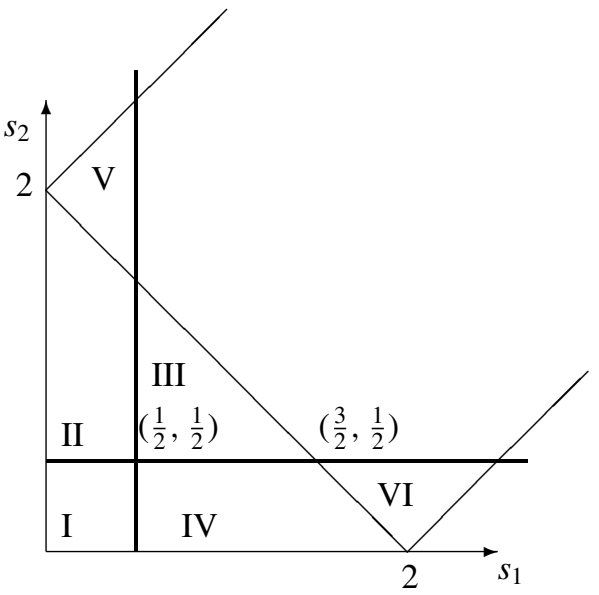

Figure 2 
(i) First we consider the open regions in the figure. Only for $\left(s_{1}, s_{2}\right)$ lying in the (open) regions I or III is it the case that $\pi$ is unitarizable (irreducible) nontempered representation.

(ii) Now we consider the boundaries. Besides the square-integrable and tempered subquotients, the other unitarizable subquotients that appear for $\left(s_{1}, s_{2}\right)$ lying on the boundaries are the subquotients of $\pi$ for $\left(s_{1}, s_{2}\right)$ on the boundary of regions I and III and $L\left(\chi_{1} v^{5 / 2}, \chi_{1} v^{1 / 2} ; 1\right)$.

Proof. As in the previous proposition, we obtain the unitarizability of region I. Consider $\chi_{1} v^{s_{1}} \times \chi_{1} v^{-s_{1}}$ in $\operatorname{GL}(2, D)$ for $s_{1} \in(0,1)$. These (irreducible) representations belong to the complementary series of $\operatorname{GL}(2, D)$, so all the subquotients of the representation $\chi_{1} v^{s_{1}} \times \chi_{1} v^{s_{1}} \rtimes 1$ are unitarizable. In the open region III this gives unitarizability of some representations and the unitarizability on the entire region then follows. This gives, by [Miličić 1973], the unitarizability of all subquotients for $\pi$ from the boundaries of I and III. Now, consider the representation $\chi_{1} v^{5 / 2} \times \chi_{1} v^{1 / 2} \rtimes 1$. If we prove that it has some nonunitarizable subquotients, this would imply the nonunitarizability in the open region VI (and, symmetrically, in $V)$. We have

$$
\begin{aligned}
\chi_{1} v^{5 / 2} \times \chi_{1} v^{1 / 2} \rtimes 1=L\left(\chi_{1} v^{3 / 2}\right. & \left.\delta\left(v, v^{-1}\right) ; 1\right)+\pi_{1} \\
& +L\left(\chi_{1} v^{5 / 2} ; \delta\left[\chi_{1} v^{1 / 2} ; 1\right]\right)+L\left(\chi_{1} v^{5 / 2}, \chi_{1} v^{1 / 2} ; 1\right) .
\end{aligned}
$$

The unitarizability of $L\left(\chi_{1} v^{5 / 2}, \chi_{1} v^{1 / 2} ; 1\right)$ is proved using global methods [Grbac 2004]. In the case $\chi_{1}=1, L\left(\chi_{1} v^{5 / 2}, \chi_{1} v^{1 / 2} ; 1\right)$ is a trivial character, so we know from [Casselman 1981] that the only unitarizable subquotients of $v^{5 / 2} \times v^{1 / 2} \rtimes 1$ are the Steinberg representation and the trivial one. In general, the nonunitarizability of $L\left(\chi_{1} v^{3 / 2} \delta\left(v, v^{-1}\right) ; 1\right)$ and $L\left(\chi_{1} v^{5 / 2} ; \delta\left[\chi_{1} v^{1 / 2} ; 1\right]\right)$ can be proved using the HoweMoore theorem [Borel and Wallach 2000]. Denote $L\left(\chi_{1} v^{3 / 2} \delta\left(v, v^{-1}\right) ; 1\right)$ by $\pi$. Consider the unbounded set $S=\left\{a_{0} \in A_{0}:\left|\alpha_{1}\left(a_{0}\right)\right|_{F} \leq 1,\left|\alpha_{2}\left(a_{0}\right)\right|=1\right\}$. We have

$$
s_{(1,1)}(\pi)=\chi_{1} v^{1 / 2} \otimes \chi_{1} v^{5 / 2}+\chi_{1} v^{1 / 2} \otimes \chi_{1} v^{-5 / 2}+\chi_{1} v^{-5 / 2} \otimes \chi_{1} v^{1 / 2} .
$$

Let $v$ and $\tilde{v}$ be the canonical lifts of the vectors in Jacquet modules corresponding to the last summand in this sum. There exists $\varepsilon>0$ such that for every $a_{0}$ from $A_{0}(\varepsilon)$ we have

$$
\left\langle\pi\left(a_{0}\right) v, \tilde{v}\right\rangle=\delta_{P_{0}}^{1 / 2}\left(a_{0}\right)\left(\chi_{1} v^{-5 / 2} \otimes \chi_{1} v^{1 / 2}\right)\left(a_{0}\right)\left\langle j_{P_{0}}(v), \tilde{j}_{P_{0}}(\tilde{v})\right\rangle .
$$

Here $A_{0}(\varepsilon)=\left\{a_{0} \in A_{0}:\left|\alpha_{1}\left(a_{0}\right)\right|_{F} \leq \varepsilon,\left|\alpha_{2}\left(a_{0}\right)\right|_{F} \leq \varepsilon\right\}$. The vectors $j_{P_{0}}(v)$ and $\tilde{j}_{P_{0}}(\tilde{v})$ denote the projection on the corresponding Jacquet module. We fix an element $a_{0}$ from $A_{0}(\varepsilon)$. Then $a_{0} S$ is a subset of $A_{0}(\varepsilon)$. So, we can find an unbounded sequence of elements in $a_{0} S$ that defines a sequence of matrix coefficients of $\pi$ not vanishing at infinity. By the Howe-Moore theorem $\pi$ is not a uniformly 
bounded representation, hence is not unitarizable. Quite analogously we prove the nonunitarizability of $L\left(\chi_{1} v^{5 / 2} ; \delta\left[\chi_{1} v^{1 / 2} ; 1\right]\right)$.

Now, consider the representation

$$
\chi_{1} v^{2} \times \chi_{1} \rtimes 1=L\left(\chi_{1} v \delta\left(v, v^{-1}\right) ; 1\right)+L\left(\chi_{1} v^{2}, \chi_{1} \rtimes 1\right) .
$$

We prove that both subquotients on the right are nonunitarizable. We have a holomorphic family of the irreducible hermitian representations $\chi_{1} v^{s} \delta\left(v, v^{-1}\right) \rtimes 1$ for $s \in\left[1, \frac{3}{2}\right)$, because we have a holomorphic family of nondegenerate hermitian forms obtained by standard intertwining operators on the compact picture of the representation $\chi_{1} \delta\left(v, v^{-1}\right) \rtimes 1$. If we assume unitarizability at $s=1$, this would imply unitarizability on the whole interval, and the unitarizability of all the subquotients on the edge of the interval, at $s=\frac{3}{2}$, which is false by the preceding reasoning. Analogously, the unitarizability of $L\left(\chi_{1} v^{2} ; \chi_{1} \rtimes 1\right)=\chi_{1} v L\left(v, v^{-1}\right) \rtimes 1$ would imply the unitarizability of all the subquotients of $\chi_{1} v^{3 / 2} L\left(v, v^{-1}\right) \rtimes 1$; but we have shown that this is not the case. This proves nonunitarizability on the region IV and on the remaining boundaries.

We continue with the examination of the principal series representations induced by the higher-dimensional representations of $D^{*}$. Let $\tau_{1}$ and $\tau_{2}$ be unitarizable representations of $D^{*}$ of dimension greater then 1 . Let $\pi=\tau_{1} \nu^{s_{1}} \times \tau_{2} \nu^{s_{2}} \rtimes 1$. We can assume that $s_{1} \geq s_{2} \geq 0$. The next four propositions are completely analogous to the first four propositions in the previous subsection, so we just note them.

Proposition 5.6. With the notation as above, assume that $\tau_{1} ¥ \tilde{\tau}_{1}$ and $\tau_{2} ¥ \tilde{\tau}_{2}$.

(i) If $\tau_{1} ¥ \tau_{2}$ and $\tau_{1} \nsucceq \tilde{\tau}_{2}$, then the representation $\pi$ has a hermitian subquotient if and only if $s_{1}=s_{2}=0$, and then $\pi$ is an irreducible tempered representation.

(ii) If $\tau_{1} \cong \tau_{2}$ then $\pi$ has a hermitian subquotient if and only if $s_{1}=s_{2}=0$, and then $\pi$ is an irreducible tempered representation.

(iii) If $\tau_{1} \cong \tilde{\tau}_{2}$ the representation $\pi$ has a hermitian subquotient if and only if $s_{1}=s_{2}$. In that case all of its subquotients are hermitian. For $s_{1} \in\left(0, \frac{1}{2}\right)$ the representation $L\left(\tau_{1} v^{s_{1}}, \tilde{\tau}_{1} v^{s_{1}} ; 1\right)$ is unitarizable, for $s_{1}>\frac{1}{2}$ nonunitarizable. We also get tempered subquotients for $s_{1} \in\left\{0, \frac{1}{2}\right\}$.

Proposition 5.7. Assume that $\tau_{1} \cong \tilde{\tau}_{1}$ and $\tau_{2} \nsucceq \tilde{\tau}_{2}$. Then the representation $\pi$ has a hermitian subquotient only if $s_{2}=0$. In that case, if $\omega_{\tau_{1}}=1, \pi$ has a tempered subquotient only if $s_{1}=\frac{1}{2}$ or $s_{1}=0$. On the other hand, $L\left(\tau_{1} v^{s_{1}}, \tau_{2} \rtimes 1\right)$ is unitarizable for $s_{1} \in\left(0, \frac{1}{2}\right]$, and for $s_{1}>\frac{1}{2}$ it is a hermitian nonunitarizable representation. If $\omega_{\tau_{1}} \neq 1, \pi$ has a tempered subquotient only if $s_{1}=0$; in that case $\pi$ is a sum of two nonequivalent tempered representations and $L\left(\tau_{1} v^{s_{1}}, \tau_{2} \rtimes 1\right)$ is hermitian nonunitarizable representation for every positive $s_{1}$. 
Proposition 5.8. Assume that $\tau_{1} ¥ \tilde{\tau}_{1}$ and $\tau_{2} \cong \tilde{\tau}_{2}$. Then $\pi$ has a hermitian subquotient only if $s_{1}=s_{2}=0$. In this case $\pi$ is an irreducible tempered representation or a sum of two nonequivalent tempered representations, depending on the central character of $\tau_{1}$. These tempered representations are those described in the previous proposition.

Proposition 5.9. Assume that $\tau_{1}$ and $\tau_{2}$ are nonisomorphic, unitary, self-contragredient representations. We keep our assumption $s_{1} \geq s_{2} \geq 0$. Then all the subquotients of the representation $\pi$ are hermitian and

(i) If $\omega_{\tau_{1}}=\omega_{\tau_{2}}=1$, we have the same situation as in Figure 1, $\pi$ has unitarizable subquotients only inside closed region I, the tempered (not square-integrable) subquotients appear for $\left(s_{1}, s_{2}\right) \in\left\{\left(\frac{1}{2}, 0\right),(0,0)\right\}$, and square-integrable representation appears for $\left(s_{1}, s_{2}\right)=\left(\frac{1}{2}, \frac{1}{2}\right)$.

(ii) If $\omega_{\tau_{1}}=1$ and $\omega_{\tau_{2}} \neq 1 \pi$ has unitarizable subquotients only for $s_{2}=0$ and $s_{1} \in$ $\left[0, \frac{1}{2}\right]$. Tempered (not square-integrable) representations appear for $\left(s_{1}, s_{2}\right) \in$ $\left\{\left(\frac{1}{2}, 0\right),(0,0)\right\}$.

(iii) If $\chi_{\tau_{1}} \neq 1$ and $\chi_{\tau_{2}} \neq 1 \pi$ is unitarizable only for $s_{1}=s_{2}=0$ and $\pi$ (as we have already seen) is a sum of 4 nonequivalent tempered representations.

Proposition 5.10. Assume that $\tau_{1} \cong \tau_{2}$ and $\tau_{1}$ is self-contragredient, such that $\omega_{\tau_{1}} \neq 1$. Then all the irreducible subquotients of the representation $\pi$ are hermitian, and the unitarizable subquotients appear only for $\left(s_{1}, s_{2}\right)$ from the closed region I on Figure 3 (when all of them are unitarizable). The square-integrable subquotients appear for $\left(s_{1}, s_{2}\right)=(1,0)$ and the tempered (not square-integrable) subquotients appear for $\left(s_{1}, s_{2}\right)=(0,0)$.

In the next proposition, we note an occurrence of the isolated unitary representation in the unitary dual, namely, the representation $L\left(\tau_{1} v^{3 / 2}, \tau_{1} v^{1 / 2} ; 1\right)$.

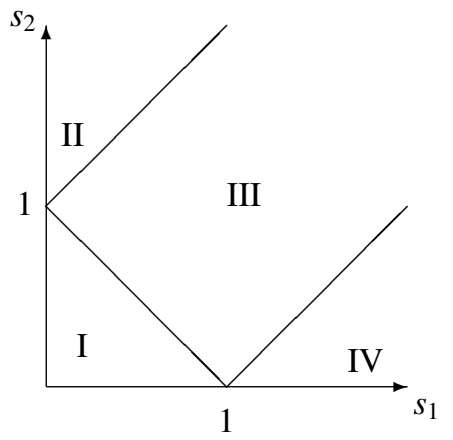

Figure 3 


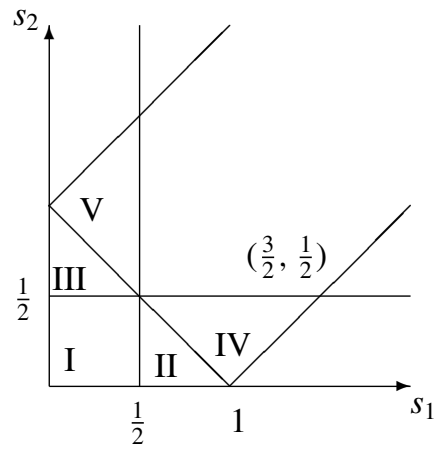

Figure 4

Proposition 5.11. We keep all the assumptions of the previous proposition, except now $\omega_{\tau_{1}}=1$. In Figure 4, considering the open regions, we have unitarizability only on region I, where we have nontempered representations. On the boundaries, we have a square-integrable subquotient for $\left(s_{1}, s_{2}\right)=\left(\frac{3}{2}, \frac{1}{2}\right)$, and tempered subquotients for $\left(s_{1}, s_{2}\right) \in\left\{\left(\frac{1}{2}, 0\right),\left(\frac{1}{2}, \frac{1}{2}\right),(0,0)\right\}$. We have other unitary subquotients on the boundary of region $I$, and $L\left(\tau_{1} v^{3 / 2}, \tau_{1} v^{1 / 2} ; 1\right)$ is a unitarizable subquotient.

Proof. We discuss only the more difficult cases. Consider the representation

$$
\begin{aligned}
& \tau_{1} v^{3 / 2} \times \tau_{1} v^{1 / 2} \rtimes 1 \\
& \quad=L\left(v \delta\left(\tau_{1} v^{1 / 2}, \tau_{1} v^{-1 / 2}\right) ; 1\right)+\pi_{4}+L\left(\tau v^{3 / 2} ; \delta\left[\tau_{1} v^{1 / 2} ; 1\right]\right)+L\left(\tau_{1} v^{3 / 2}, \tau_{1} v^{1 / 2} ; 1\right) .
\end{aligned}
$$

The unitarizability of the representation $L\left(\tau_{1} v^{3 / 2}, \tau_{1} v^{1 / 2} ; 1\right)$ is proved by global methods [Grbac 2004]. This is an isolated unitary representation in the unitary dual. We will prove the nonunitarizability of $L\left(\nu \delta\left(\tau_{1} v^{1 / 2}, \tau_{1} v^{-1 / 2}\right) ; 1\right)$ and of $L\left(\tau v^{3 / 2} ; \delta\left[\tau_{1} v^{1 / 2} ; 1\right]\right)$. We will do that in the following way: we will calculate the Plancherel measure $\mu\left(s, \delta\left(\tau_{1} v^{1 / 2}, \tau_{1} v^{-1 / 2}\right)\right)$. Let us again denote by $A(s)$ the standard intertwining operator such that

$$
A(s): \delta\left(\tau_{1} v^{1 / 2}, \tau_{1} v^{-1 / 2}\right) v^{s} \rtimes 1 \rightarrow \delta\left(\tau_{1} v^{1 / 2}, \tau_{1} v^{-1 / 2}\right) v^{-s} \rtimes 1 .
$$

We will prove that the Plancherel measure has a simple pole at $s=1$ and that $A(s)$ has no pole at $s=-1$. We will apply these observations to the calculation of Jantzen filtrations near $s=1$. This will give us nonunitarizability. Let $\delta=\delta\left(\tau_{1} v^{1 / 2}, \tau_{1} v^{-1 / 2}\right)$. First, we use the previously mentioned result stating that $\mu(s, \delta)=\mu\left(s, \delta^{\prime}\right)$. The representation $\delta^{\prime}$ is generic, so we can apply the results from [Shahidi 1990b] to compute the Plancherel measure in terms of the $\gamma$-factors. Up to an exponential factor, we have

$$
\mu\left(\frac{s}{2}, \delta^{\prime}\right) \approx \frac{\gamma\left(s, \delta^{\prime}, \Lambda^{2} \rho_{4}, \psi\right)}{\gamma\left(1+s, \delta^{\prime}, \Lambda^{2} \rho_{4}, \psi\right)},
$$


where $\psi$ is a nontrivial additive character of $F$, given in advance. By multiplicativity of $\gamma$-factors [Shahidi 1990a] we have

$$
\begin{array}{r}
\gamma\left(s, \delta^{\prime}, \Lambda^{2} \rho_{4}, \psi\right) \\
=\gamma\left(s, \tau_{1} v^{1 / 2}, \operatorname{det}, \psi\right) \gamma\left(s, \tau_{1} v^{-1 / 2}, \operatorname{det}, \psi\right) \gamma\left(s, \tau_{1} v^{1 / 2} \times \tau_{1} v^{1 / 2}, \psi\right) .
\end{array}
$$

The last factor is a Rankin-Selberg $\gamma$-factor. When a $\gamma$-factor is expressed in terms of $L$-functions, we obtain, up to an exponential factor,

$$
\mu\left(\frac{s}{2}, \delta^{\prime}\right) \approx \frac{\left(1-q^{-1-s}\right)\left(1-q^{1-s}\right)\left(1-q^{1+s}\right)\left(1-q^{-1+s}\right)\left(1-q^{-r s}\right)\left(1-q^{r s}\right)}{\left(1-q^{s}\right)\left(1-q^{-s}\right)\left(1-q^{-2+s}\right)\left(1-q^{-2-s}\right)\left(1-q^{-r+r s}\right)\left(1-q^{-r-r s}\right)},
$$

where $r$ is a natural number satisfying $L\left(s, \tau_{1} \times \tau_{1}\right)=\left(1-q^{r s}\right)^{-1}$. Indeed $\mu\left(s, \delta^{\prime}\right)$ has a simple pole at $s=1$. Denote by $w_{\varepsilon}$ the reflection in the Weyl group with respect to the root $\varepsilon$. Then consider the intertwining operator

$$
A_{w_{\alpha+\beta}}(s): \tau_{1} v^{s+\frac{1}{2}} \times \tau_{1} v^{s-\frac{1}{2}} \rtimes 1 \rightarrow \tau_{1} v^{-s+\frac{1}{2}} \times \tau_{1} v^{-s-\frac{1}{2}} \rtimes 1 .
$$

The poles of the operator $A(s)$ are among the poles of the operator $A_{w_{\alpha+\beta}}(s)$ and $\left.A_{w_{\alpha+\beta}}(s)\right|_{\delta v^{s} \rtimes 1}=A(s)$. But using the factorization of the operator $A_{w_{\alpha+\beta}}(s)$ [Shahidi 1981], we see that it has no poles at $s=-1$. Let $X$ denotes the compact picture of the representation $\delta v^{s} \rtimes 1$. We will consider the Jantzen filtrations of the space $X$, for $s \in[0,1]$. For $s \in(0,1)$ the representations $\delta v^{s} \rtimes 1$ are irreducible, and the mentioned interval parameterizes a nondegenerate family of hermitian forms in the compact picture $X$. For $\mathrm{s}=0, A(s)$ is holomorphic, and, normalized, generates the intertwining algebra of the representation $\delta \rtimes 1=T_{1}+T_{2}$ (follows from the proof of the Proposition 2.5). The operator $A(0)$ endows the space of this representation with the hermitian form which is of a different sign on each of the $T_{i}$ 's. This gives us the nonunitarizability of $\delta v^{s} \rtimes 1$ for $s \in(0,1)$. By the theory of Jantzen filtrations [Vogan 1984], at $s=1$ we consider filtrations $X=X_{1}^{0} \supset X_{1}^{1} \supset \cdots \supset 0$. Because we have a standard representation, we have $X_{1}^{1}=\pi_{4}$, a square-integrable representation. We will prove that $X_{1}^{2}=\{0\}$, i.e., that a hermitian form defined on $X_{1}^{1}$ by

$$
<v, v^{\prime}>_{1}=\lim _{s \rightarrow 1} \int_{K}<v(k), \frac{1}{s-1} A(s) v_{s}^{\prime}(k)>d k
$$

is nondegenerate, so its radical, namely $X_{1}^{2}$, is trivial. Because of the simplicity of the pole of the Plancherel measure at $s=1$, we have

$$
A(-s) \frac{1}{s-1} A(s)=h(s),
$$

where $h$ is holomorphic function in the neighborhood of $s=1$, and $h(1) \neq 0$. Hence, for nonzero $v^{\prime} \in X$ such that $v_{1}^{\prime} \in \pi_{4}$, we have $\lim _{s \rightarrow 1} A(s) v_{s}^{\prime} /(s-1) \notin L(\delta v, 1)$. 
Now, we can choose $v \in X_{1}^{1}$ such that

$$
\left\langle v, v^{\prime}\right\rangle_{1}=\lim _{s \rightarrow 1} \int_{K}\left\langle v(k), \frac{A(s) v_{s}^{\prime}(k)}{s-1}\right\rangle d k \neq 0 .
$$

We can obtain the signature of $\delta v^{s} \rtimes 1$ for $s>1$ and for $s<1$ in terms of signatures $\left(p_{0}, q_{0}\right)$ and $\left(p_{1}, q_{1}\right)$. But we know that on these segments we have nonunitarizable representations. We conclude that $p_{0} \neq 0$ and $q_{0} \neq 0$, which is equivalent to nonunitarizability.

The proof of the nonunitarizability of $L\left(\tau_{1} v^{3 / 2} ; \delta\left[\tau_{1} v^{1 / 2} ; 1\right]\right)$ follows the same pattern: We will compute the Plancherel measure of $\mu\left(s, \tau_{1} \otimes \delta\left[\tau_{1} \nu^{s} ; 1\right]\right)$. We can easily extend the results from the fourth section to the case when we consider square-integrable representations instead of cuspidal ones. So we have

$$
\mu\left(s, \tau_{1} \otimes \delta\left[\tau_{1} v^{s} ; 1\right]\right)^{2}=\mu\left(s, \tau_{1}^{\prime} \otimes \sigma_{1}\right) \mu\left(s, \tau_{1}^{\prime} \otimes \sigma_{2}\right) .
$$

The representations $\sigma_{i}, i=1,2$, from the above equation are obtained originally by considering the restrictions of the representations to the groups $\operatorname{SL}(2, F) D_{1}$ or $\operatorname{SL}(2, F) \cdot \operatorname{SL}(2, F)$. It is not hard to see that, in this case, $\sigma_{i} \hookrightarrow \operatorname{Ind}_{M_{\alpha}}^{\operatorname{SO}(4, F)}\left(\tau_{1}^{\prime} \chi_{i}\right)$, where $\chi_{i}$ is a quadratic character on $F^{*}$. Here $M_{\alpha}$ is the standard Levi subgroup, which is diagonally positioned in $\mathrm{SO}(4, F)$, and $\tau_{1}^{\prime}$ is a Langlands' lift of the representation $\tau_{1}$. Because of the genericity of the representations $\sigma_{i}$, we can apply the results of Shahidi about multiplicativity of $\gamma$-factors. We obtain that the Plancherel measure $\mu\left(s, \tau_{1}^{\prime} \otimes \sigma_{i}\right)$ can have a pole of order one at $s=\frac{3}{2}$. We obtain a pole there if and only if, $\tau_{1}^{\prime} \cong \tau_{1}^{\prime} \chi_{i}$. But $\mu\left(s, \tau_{1} \otimes \delta\left[\tau_{1} v^{1 / 2} ; 1\right]\right)$ must have a pole there, so it is a pole of order one. As in the previous case, we conclude that the intertwining operators appearing in the definition of the Plancherel measure $\mu\left(s, \tau_{1} \otimes \delta\left[\tau_{1} v^{s} ; 1\right]\right)$ are holomorphic near $s= \pm \frac{3}{2}$. Now we can conclude, as in the previous discussion, that $L\left(\tau_{1} v^{3 / 2} ; \delta\left[\tau_{1} v^{1 / 2} ; 1\right]\right)$ is a nonunitarizable representation. The only Langlands quotient left to settle is $L\left(\tau_{1} v ; \tau_{1} \rtimes 1\right)=v^{1 / 2} L\left(\tau_{1} v^{1 / 2}, \tau_{1} v^{-1 / 2}\right) \rtimes 1$. We obtain the hermiticity of the representations $\pi_{s}=v^{s} L\left(\tau_{1} v^{1 / 2}, \tau_{1} v^{-1 / 2}\right) \rtimes 1$ for $s \in(0,1)$ using the action of the long intertwining operator acting on the space $\tau_{1} v^{s+\frac{1}{2}} \times \tau_{1} v^{s-\frac{1}{2}} \rtimes 1$. But unitarity of the representation $\pi_{s}$ at $s=\frac{1}{2}$ would imply unitarizability of all the subquotients at $s=1$, which contradicts what we have just proved.

Again, let $\pi=\tau_{1} v^{s_{1}} \times \chi_{1} \nu^{s_{2}} \rtimes 1$. We can assume $s_{1}, s_{2} \geq 0$. The proof of the next result is straightforward.

Proposition 5.12. (i) If $\tau_{1}$ is not a self-contragredient representation and $\chi_{1}^{2} \neq 1$, $\pi$ has a hermitian quotient only if $\left(s_{1}, s_{2}\right)=(0,0)$, and then $\pi$ is an irreducible, tempered representation. 
(ii) If $\tau_{1}$ is selfdual, but $\chi_{1}^{2} \neq 1 \pi$ has a hermitian quotient only if $s_{2}=0$ (then all the subquotients are hermitian), and has unitarizable subquotients for $s_{1}$ from the segment $\left[0, \frac{1}{2}\right]$ if $\omega_{\tau_{1}}=1$ and only in the origin if $\omega_{\tau_{1}} \neq 1$. If $\omega_{\tau_{1}}=1, \pi$ is irreducible tempered for $s_{1}=0$, and has a tempered subquotient for $s_{1}=\frac{1}{2}$. If $\omega_{\tau_{1}} \neq 1$, we obtain a tempered representation for $s_{1}=s_{2}=0$.

(iii) If $\chi_{1}^{2}=1$ and $\tau_{1}$ is not a selfdual representation, $\pi$ has a hermitian subquotient only for $s_{1}=0$ (then all quotients are hermitian). For $s_{2}=0$ representation $\pi$ is irreducible tempered, for $s_{2}=\frac{1}{2}$ it has a tempered subquotient, and unitarizable subquotients appear for $\left\{\left(0, s_{2}\right): s_{2} \in\left[0, \frac{1}{2}\right]\right\}$.

(iv) If $\chi_{1}^{2}=1$ and $\tau_{1} \rtimes 1$ reduces, every subquotient of $\pi$ is hermitian. For $s_{1}=s_{2}=0$ representation $\pi$ is a sum of two-nonequivalent tempered representations, and for $\left(s_{1}, s_{2}\right)=\left(0, \frac{1}{2}\right)$ has a tempered subquotient. Other unitarizable subquotients appear for $s_{1}=0$ and $s_{2} \in\left[0, \frac{1}{2}\right]$.

(iv) If $\chi_{1}^{2}=1$ and $\tau_{1} v^{1 / 2} \rtimes 1$ reduces, we have the analogous situation as for the characters; unitarizability of all subquotients of $\pi$ on the closed region I in Figure 1 .

\subsection{The unitary dual supported on the nonminimal parabolic subgroups. Once} we have handled the reducibility questions in this case, the rest is straightforward. Assume that $s \geq 0$.

Proposition 5.13. (i) We consider induction from the Siegel parabolic subgroup: If $\tau$ is an irreducible cuspidal representation of $\operatorname{GL}(2, D)$, let $\pi_{s}=\tau \nu^{s} \rtimes 1$. If $\tau$ is not self-dual, $\pi_{s}$ is hermitian only when $s=0$; then it is a tempered representation. If $\tau$ is self-dual, $\pi_{s}$ reduces for some $s_{0} \in\left\{0, \frac{1}{2}\right\}$, and all of it subquotients are always hermitian. In this case, if $s_{0}=0, \pi_{0}$ is the sum of two nonequivalent tempered representations, and otherwise, $\pi_{s}$ are nonunitarizable. If $s_{0}=\frac{1}{2}, \pi_{s}$ is a nontempered irreducible unitary representation for $s \in\left(0, \frac{1}{2}\right)$, tempered for $s=0$, and for $s=\frac{1}{2}$ the representation $\pi_{\frac{1}{2}}$ has two irreducible unitary subquotients; one of them is nontempered and the other is a square-integrable representation.

(ii) We consider induction from the non-Siegel parabolic subgroup: the representation $\tau \nu^{s} \rtimes \delta$ for irreducible representation $\tau$ of the group $D^{*}$ and irreducible cuspidal representation $\delta$ of the group $G_{1}(D)$ is unitarizable for nonselfdual $\tau$ only for $s=0$, and then it is irreducible tempered representation. Otherwise, it reduces for some $s_{0} \in\left\{0, \frac{1}{2}, 1, \frac{3}{2}\right\}$ (Corollaries 4.2 and 4.3). If $s_{0}=0, \pi_{0}$ is a sum of two nonequivalent tempered representations, otherwise $\pi_{s}$ is nonunitarizable. If $s_{0} \in\left\{\frac{1}{2}, 1, \frac{3}{2}\right\}$, the representation $\pi_{0}$ is tempered, nontempered unitarizable for $s \in\left(0, s_{0}\right)$ and for $s=s_{0}$ it has a nontempered unitarizable Langlands quotient, and a square-integrable subrepresentation. 
Acknowledgments. I express my gratitude to Profs. M. Tadić and G. Muić for their constant help, and to my colleague N. Grbac for helpful conversations.

\section{References}

[Aubert 1995] A.-M. Aubert, "Dualité dans le groupe de Grothendieck de la catégorie des représentations lisses de longueur finie d'un groupe réductif $p$-adique", Trans. Amer. Math. Soc. 347:6 (1995), 2179-2189. Erratum in 347:6 (1995), 2179-2189. MR 95i:22025 Zbl 0827.22005

[Ban 1999] D. Ban, "Parabolic induction and Jacquet modules of representations of O $(2 n, F)$ ", Glas. Mat. Ser. III 34:2 (1999), 147-185. MR 2001m:22033 Zbl 0954.22013

[Bernstein et al. 1984] J. Bernstein, P. Deligne, D. Kazhdan, and M.-F. Vignéras, Représentations des groupes réductifs sur un corps local, Travaux en Cours, Hermann, Paris, 1984. MR MR771670 (85h:22001) Zbl 0544.00007

[Borel and Wallach 2000] A. Borel and N. Wallach, Continuous cohomology, discrete subgroups, and representations of reductive groups, 2nd ed., Mathematical Surveys and Monographs 67, American Mathematical Society, Providence, RI, 2000. MR 2000j:22015 Zbl 0980.22015

[Casselman 1981] W. Casselman, "A new nonunitarity argument for $p$-adic representations", J. Fac. Sci. Univ. Tokyo Sect. IA Math. 28:3 (1981), 907-928. MR 84e:22018 Zbl 0519.22011

[Casselman 1995] W. Casselman, "Introduction to the theory of admissible representations of $p$-adic reductive groups", preprint, 1995, Available at www.math.ubc.ca/ cass/research/p-adic-book.dvi.

[Flicker 1987] Y. Z. Flicker, "Rigidity for automorphic forms", J. Analyse Math. 49 (1987), 135202. MR 89a:11057 Zbl 0656.10024

[Flicker 1992] Y. Z. Flicker, "On the symmetric square: applications of a trace formula", Trans. Amer. Math. Soc. 330:1 (1992), 125-152. MR 92g:11052 Zbl 0761.11027

[Flicker and Kazhdan 1988] Y. Z. Flicker and D. A. Kazhdan, "A simple trace formula", J. Analyse Math. 50 (1988), 189-200. MR 90e:11078 Zbl 0666.10018

[Goldberg and Shahidi 2001] D. Goldberg and F. Shahidi, "On the tempered spectrum of quasi-split classical groups. II”, Canad. J. Math. 53:2 (2001), 244-277. MR 2001m:22035 Zbl 0964.22014

[Grbac 2004] N. Grbac, "The residual spectrum of the Hermitian quaternionic group of the split rank 2", preprint, Faculty of Electrical Enginereeing and Computing, University of Zagreb, 2004.

[Hanzer 2004] M. Hanzer, " $R$ groups for quaternionic Hermitian groups", Glas. Mat. Ser. III 39:1 (2004), 31-48. MR 2005d:22008 Zbl 1056.22010

[Konno 2001] K. Konno, "Induced representations of U(2,2) over a p-adic field", J. Reine Angew. Math. 540 (2001), 167-204. MR 2003a:22016 Zbl 0982.22011

[Labesse and Langlands 1979] J.-P. Labesse and R. P. Langlands, " $L$-indistinguishability for SL(2)”, Canad. J. Math. 31:4 (1979), 726-785. MR 81b:22017 Zbl 0421.12014

[Miličić 1973] D. Miličić, “On $C^{*}$-algebras with bounded trace”, Glasnik Mat. Ser. III 8 (1973), 7-22. MR 48 \#2781

[Mœglin and Waldspurger 1995] C. Mœglin and J.-L. Waldspurger, Spectral decomposition and Eisenstein series, Cambridge Tracts in Mathematics 113, Cambridge University Press, Cambridge, 1995. MR 97d:11083 Zbl 0846.11032

[Mœglin et al. 1987] C. Mœglin, M.-F. Vignéras, and J.-L. Waldspurger, Correspondances de Howe sur un corps p-adique, Lecture Notes in Mathematics 1291, Springer, Berlin, 1987. MR 91f:11040 Zbl 0642.22002

[Muić 1997] G. Muić, “The unitary dual of p-adic G2”, Duke Math. J. 90:3 (1997), 465-493. MR 98k:22073 Zbl 0896.22006 
[Muić and Savin 2000] G. Muić and G. Savin, "Complementary series for Hermitian quaternionic groups”, Canad. Math. Bull. 43:1 (2000), 90-99. MR 2001g:22019 Zbl 0945.22005

[Sally and Tadić 1993] P. J. Sally, Jr. and M. Tadić, "Induced representations and classifications for $\operatorname{GSp}(2, F)$ and $\operatorname{Sp}(2, F)$ ", Mém. Soc. Math. France (N.S.) 52 (1993), 75-133. MR 94e:22030 Zbl 0784.22008

[Shahidi 1981] F. Shahidi, “On certain L-functions”, Amer. J. Math. 103:2 (1981), 297-355. MR 82i: 10030 Zbl 0467.12013

[Shahidi 1990a] F. Shahidi, "On multiplicativity of local factors", pp. 279-289 in Festschrift in honor of I. I. Piatetski-Shapiro on the occasion of his sixtieth birthday (Ramat Aviv, 1989), vol. II, edited by S. Gelbart et al., Israel Math. Conf. Proc. 3, Weizmann, Jerusalem, 1990. MR 93e:11144 Zbl 0841.11061

[Shahidi 1990b] F. Shahidi, “A proof of Langlands' conjecture on Plancherel measures; complementary series for p-adic groups", Ann. of Math. (2) 132:2 (1990), 273-330. MR 91m:11095 Zbl 0780.22005

[Shahidi 1992] F. Shahidi, "Twisted endoscopy and reducibility of induced representations for $p$ adic groups”, Duke Math. J. 66:1 (1992), 1-41. MR 93b:22034 Zbl 0785.22022

[Silberger 1980] A. J. Silberger, "Special representations of reductive $p$-adic groups are not integrable”, Ann. of Math. (2) 111:3 (1980), 571-587. MR 82k:22015 Zbl 0437.22015

[Speh and Vogan 1980] B. Speh and D. A. Vogan, Jr., "Reducibility of generalized principal series representations", Acta Math. 145:3-4 (1980), 227-299. MR 82c:22018 Zbl 0457.22011

[Tadić 1983] M. Tadić, "The topology of the dual space of a reductive group over a local field", Glas. Mat. Ser. III 18:2 (1983), 259-279. MR 85k:22043 Zbl 0536.22026

[Tadić 1990] M. Tadić, "Induced representations of $\operatorname{GL}(n, A)$ for $p$-adic division algebras $A$ ", $J$. Reine Angew. Math. 405 (1990), 48-77. MR 91i:22025 Zbl 0684.22008

[Tadić 1994] M. Tadić, "Representations of p-adic symplectic groups", Compositio Math. 90:2 (1994), 123-181. MR 95a:22025 Zbl 0797.22008

[Tadić 1995] M. Tadić, "Structure arising from induction and Jacquet modules of representations of classical p-adic groups”, J. Algebra 177:1 (1995), 1-33. Zbl 0874.22014

[Tadić 1998] M. Tadić, “On reducibility of parabolic induction”, Israel J. Math. 107 (1998), 29-91. MR 2001d:22012 Zbl 0914.22019

[Vogan 1984] D. A. Vogan, Jr., "Unitarizability of certain series of representations", Ann. of Math. (2) 120:1 (1984), 141-187. MR 86h:22028 Zbl 0561.22010

[Weil 1973] A. Weil, Basic number theory, 2nd ed., Grundlehren der Math. Wissenschaften 144, Springer, Berlin, 1973. MR 96c:11002 Zbl 0267.12001

[Zelevinsky 1981] A. V. Zelevinsky, Representations of finite classical groups: a Hopf algebra approach, Lecture Notes in Mathematics 869, Springer, Berlin, 1981. MR 83k:20017 Zbl 0465.20009

Received October 5, 2004. Revised May 17, 2005.

\section{MARCELA HANZER}

DEPARTMENT OF MATHEMATICS

UNIVERSITY OF ZAGREB

BIJENICKA 30

10000 ZAGREB

CROATIA

hanmar@math.hr 